\title{
Extracting a Largest Redundancy-Free XML Storage Structure from an Acyclic Hypergraph in Polynomial Time
}

\author{
Wai Yin Mok, Joseph Fong ${ }^{\dagger}$ and David W. Embley
}

\begin{abstract}
Given a hypergraph and a set of embedded functional dependencies, we investigate the problem of determining the conditions under which we can efficiently generate redundancy-free XML storage structures with as few scheme trees as possible. Redundancy-free XML structures guarantee both economy in storage space and the absence of update anomalies, and having the least number of scheme trees requires the fewest number of joins to navigate among the data elements. We know that the general problem is intractable. The problem may still be intractable even when the hypergraph is acyclic and each hyperedge is in Boyce-Codd Normal Form (BCNF). As we show here, however, given an acyclic hypergraph with each hyperedge in BCNF, a polynomial-time algorithm exists that generates a largest possible redundancyfree XML storage structure. Successively generating largest possible scheme trees from among hyperedges not already included in generated scheme trees constitutes a reasonable heuristic for finding the fewest possible scheme trees. For many practical cases, this heuristic finds the set of redundancy-free XML storage structures with the fewest number of scheme trees. In addition to a correctness proof and a complexity analysis showing that the algorithm is polynomial, we also give experimental results over randomly generated but appropriately constrained hypergraphs showing empirically that the algorithm is indeed polynomial.
\end{abstract}

Keywords: XML data redundancy, large XML storage structures, XML-Schema generation, acyclic hypergraphs

\section{Introduction}

XML databases are emerging [5]. Two types of XML databases are native XML databases, whose backend storage structures are internal representations of XML documents, and XML-enabled databases, whose backend storage structures are internal representations of relational tables. The fundamental unit of (logical) storage in native XML databases is an XML document [4]. Thus, designing XML documents for efficient retrieval and update has been a topic of recent research

\footnotetext{
${ }^{*}$ Department of Economics and Information Systems, University of Alabama in Huntsville, Huntsville, Alabama 35899, USA, mokw@email.uah.edu. (Most of this research was conducted while W. Y. Mok was a Visiting Research Fellow at City University of Hong Kong.)

${ }^{\dagger}$ Department of Computer Science, City University of Hong Kong, Hong Kong, China, csjfong@cityu.edu.hk.

${ }^{\ddagger}$ Department of Computer Science, Brigham Young University, Provo, Utah 84602, USA, embley@cs.byu.edu.
} 
$[9,11,12]$. The fundamental unit of (logical) storage in XML-enabled databases is a relational table. This table-storage method requires various mapping rules to translate between XML document schemas and database schemas and employs middleware to transfer data between XML documents and databases $[4,20,23]$. A recent study shows that designing XML documents for efficient retrieval and update can also guarantee well-designed relational storage structures for XML-enabled databases [13]. Thus, for both native XML databases and XML-enabled databases, designing XML documents for efficient retrieval and update is an appropriate focus for study.

Similar to design of relational tables by normalizing relational schemas, designing XML documents for efficient retrieval and update is about normalizing XML storage schemas. Normalized XML storage schemas remove the possibility of redundancy with respect to constraints and typically make both retrieval and update more efficient. Thus, there has been a flurry of research work on normalization of XML documents $[2,6,7,15,18,22,25,26,27]$.

This paper, which follows up on our previous work [7, 18], is another step in this direction. In [18] we showed that generating a minimum number of redundancy-free XML storage structures from a conceptual-model hypergraph is NP-hard. Here we consider special-case conditions ${ }^{1}$ that commonly hold in practice in an effort to find an efficient algorithm. Since it is known that checking whether relational schemas are in Boyce-Code Normal Form (BCNF) is intractable, our first condition limits conceptual-model hypergraphs to those in which each hypergraph edge is in BCNF with respect to the given functional dependencies (FDs). Next, since cycles in hypergraphs introduce ambiguity and typically cause difficulties, we assume that conceptual-model hypergraphs are acyclic. Finally, we assume that the only multivalued dependencies (MVDs) are hypergraphgenerated MVDs. Even with these assumptions, however, it is an open problem to find an algorithm that generates a minimum number of redundancy-free XML storage structures in polynomial time. We therefore settle on a heuristic that resolves the issue for many practical cases and likely gives good results for all cases.

As the basis of our heuristic, we provide in this paper a polynomial-time algorithm that generates a largest scheme tree from an acyclic hypergraph and a set of FDs where each FD is embedded in some hyperedge and each hyperedge is in BCNF. As an approximation to generating a minimum number of redundancy-free XML storage structures, we use this heuristic repeatedly on the remaining hypergraph edges not already included in generated scheme-tree storage structures. This heuristic always yields redundancy-free XML storage structures and often, especially in practical cases, yields the fewest.

To illustrate or our approach and to show some of the pitfalls involved, we present a motivating example. In this example, we rely on intuition for some undefined terms. Later in Section 2, we

\footnotetext{
${ }^{1}$ In making these special-case assumptions, we point out that many conceptual-model hypergraphs found in practice satisfy these assumptions without any need for modification. For those that do require some modification to satisfy these conditions, the modifications are often minimal and straightforward. (1) In practice, conceptual-model hypergraph edges rarely violate BCNF. Further, since the size of a edge is typically small, checking exhaustively for keys of the edge and for applicable non-trivial FDs is not inordinately expensive. (2) In practice, we can always introduce role attributes, as needed, to break cycles. (3) In practice, we almost never care about any MVDs except hypergraph-generated MVDs.
} 


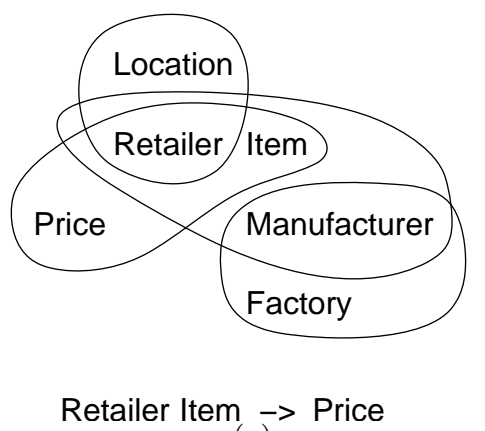

(a)

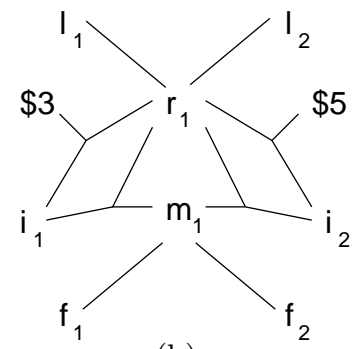

(b)

Figure 1: The Acyclic Hypergraph and Relationships of Example 1.

formally define these terms.

Example 1 Figure 1(a) shows an acyclic hypergraph and an FD, Retailer Item $\rightarrow$ Price, embedded in one of the hypergraph edges. Figure 1(b) shows some possible relationships among instance values for the hyperedges in Figure 1(a). For example, two of the relationships are "retailer $r_{1}$ sells item $i_{1}$ for $\$ 3$ " and "manufacturer $m_{1}$ has factory $f_{1}$." Figures 2(a), 2(b), and 2(c) show three possible sets of scheme trees and their associated instances taken from the relationships in Figure 1(b). In Figure 2(a), because there is only one scheme-tree instance, the data values are compactly stored. However, the instance data is redundant. Since manufacturer $m_{1}$ is necessarily stored twice, the dependent factories, which must be the same, are therefore redundantly stored more than once. In Figure 2(b), even though no data redundancy is present in any of the scheme-tree instances, there are more trees than necessary. The largest redundancy-free scheme tree for this example is the one on the left in Figure 2(c), which balances the requirements of data redundancy and compactness of data. Creating this scheme tree first followed by creating a scheme tree from the remaining hyperedge $\{$ Manufacturer, Factory\} yields the fewest possible redundancy-free scheme trees.

By way of comparison with the XML normalization work of others $[2,6,15,22,25,26,27]$, we point out that our approach differs significantly. Not only have these other researchers defined their FDs, and thus their normal forms, differently, the basis of our approach is also different from theirs. As opposed to the complicated FDs defined in these papers, we rely on standard FD and hypergraph-generated MVD definitions, which can be straightforwardly derived from conceptualmodel hypergraphs. Furthermore, the basis of our approach is conceptual models, which have not been considered at all in other XML normalization work. We believe our approach is more common in practice and in line with the tradition followed by information-system developers, who first create conceptual-model instances and then generate database storage structures.

We give the details of our contribution of generating a largest possible scheme tree from a conceptual-model hypergraph in polynomial time as follows. We first lay the ground work by providing basic definitions in Section 2. Based on this foundation, we present the polynomial-time, scheme-tree generation algorithm in Section 3. Throughout Sections 2 and 3 we provide examples 

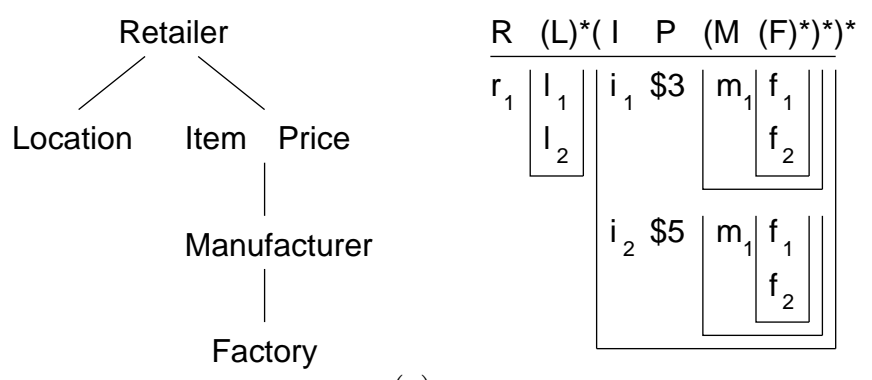

(a)
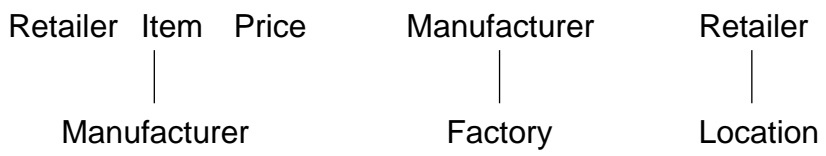

$$
\begin{aligned}
& \begin{array}{l|l|l|}
R & P & (M)^{*} \\
\hline r_{1} i_{1} & \$ 3 & m_{1} \mid
\end{array} \\
& r_{1} i_{2} \$ 5\left|m_{1}\right|
\end{aligned}
$$

(b)

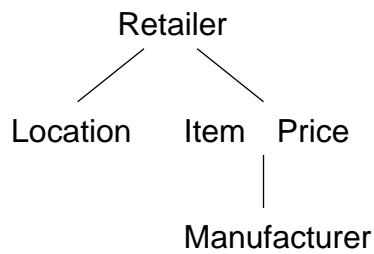

\begin{tabular}{|c|c|c|}
\hline$R \quad$ (L) & ( 1 P & $(\mathrm{M})^{*}$ \\
\hline \begin{tabular}{l|l}
$r_{1}$ & $l_{1}$
\end{tabular} & $i_{1} \$ 3$ & $m_{1}$ \\
\hline$I_{2}$ & $i_{2} \$ 5$ & $m_{1}$ \\
\hline
\end{tabular}
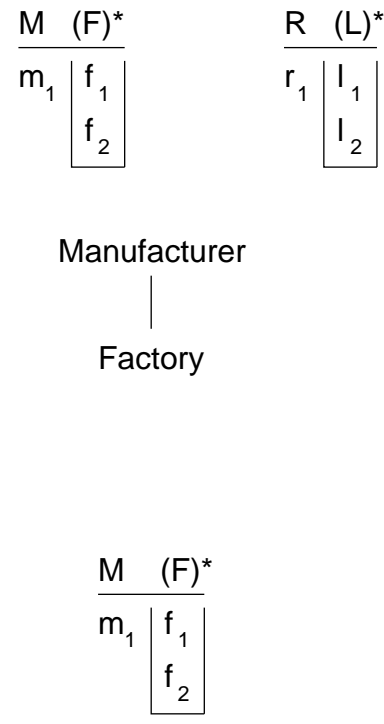

(c)

Figure 2: The Scheme Trees and Scheme-Tree Instances of Example 1.

to motivate and illustrate definitions and algorithmic procedures. We present experimental data to verify our algorithm in Section 4 and formally prove our claims in Section 5. We make concluding remarks in Section 6.

\section{Basic Definitions}

\subsection{Acyclic Hypergraphs}

To make this paper self-contained, we borrow some definitions from previous work. The first three definitions are from [3].

Definition 1 Let $U$ be a set of attributes. A hypergraph $H=\left\{E_{1}, \ldots, E_{n}\right\}$ over $U$ is a set of 
subsets of $U$ where each subset $E_{i}$ is called a hyperedge of $H$, or simply an edge of $H$ if the context is clear.

Definition 2 Graham Reduction, also known as GYO Reduction [10], applies two operations to a hypergraph $H=\left\{E_{1}, \ldots, E_{n}\right\}(n \geq 1)$ until neither can be applied. These two operations are: (Attribute Removal) If $A$ is an attribute that appears in exactly one edge $E_{i}$, then delete $A$ from $E_{i}$. (Edge Removal) Delete an edge $E_{i}$ if there is an edge $E_{j}$ such that $i \neq j$ and $E_{i} \subseteq E_{j}$.

Definition 3 A hypergraph is acyclic if Graham Reduction reduces it to the empty set.

Definition 4 A hypergraph is reduced if none of its hyperedges is a proper subset of another hyperedge.

By repeatedly applying the edge-removal step of Graham Reduction, it is easy to observe that a hypergraph is acyclic if and only if its reduced form is acyclic. All hypergraphs considered in this paper are assumed to be reduced.

We now introduce a procedure that makes use of Graham Reduction to create a data structure from a reduced acyclic hypergraph called a join tree.

\section{Procedure CreateJoinTree}

Input: a reduced acyclic hypergraph $H$.

Output: a join tree $T$ for $H$, and a set of labels for $H$.

1. Initially, let $T$ be a graph with no edges whose nodes are the unique hyperedges in $H$.

2. Apply Graham Reduction: while applying Graham Reduction, when a remaining hyperedge $E_{i}^{\prime}$, which is the result of applying one or more attribute removals to an original hyperedge $E_{i}$, is removed because it is a subset of an original hyperedge $E_{j}$, create an edge $\left\{E_{i}, E_{j}\right\}$ for $T$ and label the edge $E_{i}^{\prime}$. In the process, $E_{i}^{\prime}$ becomes a label of $H$. (Since $E_{i}^{\prime}$ may be a subset of more than one hyperedge, more than one join tree is possible for a given reduced acyclic hypergraph.)

3. When the Graham Reduction is complete, the graph $T$ will have become a join tree; thus return $T$.

Example 2 Figure 3 shows a possible join tree created by Procedure CreateJoinTree for the acyclic hypergraph in Figure 1(a). In another join tree for the hypergraph in Figure 1(a), instead of the Retailer edge between \{Retailer, Location\} and \{Retailer, Item, Price\}, the join tree can have a Retailer edge between $\{$ Retailer, Location $\}$ and $\{$ Retailer, Item, Manufacturer $\}$.

\subsection{Constraints}

In this paper, FDs and hypergraph-generated MVDs are the only constraints we consider. These are typically the most common constraints encountered in practice. FDs have their standard definition. The definition of hypergraph-generated MVDs is from [3] and [8]. 


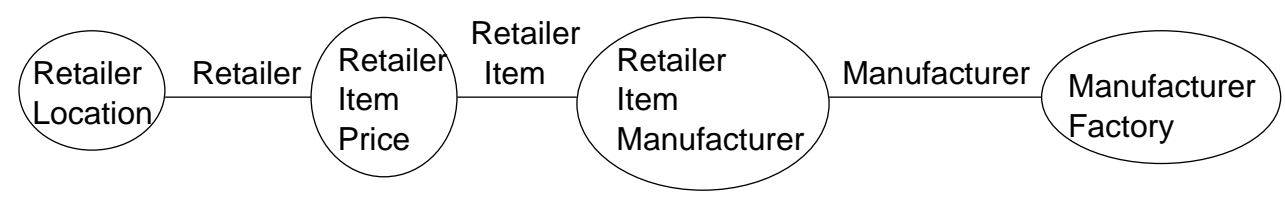

Figure 3: A Join Tree of the Acyclic Hypergraph in Figure 1(a).

Definition 5 Two hyperedges are connected if they have a nonempty intersection. A set $S$ of hyperedges is disconnected if $S$ can be partitioned into two nonempty subsets $S_{1}$ and $S_{2}$ such that no hyperedge in $S_{1}$ is connected to any hyperedge in $S_{2}$. A set of hyperedges is connected if it is not disconnected. A connected component is a maximal connected set of hyperedges. A hypergraph $H$ generates a number of MVDs of the form $X \rightarrow Y_{1}\left|Y_{2}\right| \cdots \mid Y_{n}$ where $X$ and $Y_{1}, \ldots, Y_{n}$ are disjoint sets of attributes and each $Y_{i}$ is a maximal connected set of hyperedges constructed from the hyperedges of $H$ after they have been reduced by the removal of the attributes in $X$, i.e., the maximal connected components of $\{E-X: E$ is a hyperedge of $H\}-\{\emptyset\}$.

Example 3 Removing the attributes Retailer and Item from Figure 1(a) results in the hypergraphgenerated MVDs Retailer Item $\rightarrow$ Manufacturer Factory | Price | Location. Removing Manufacturer and Factory results in the trivial MVD Manufacturer Factory $\rightarrow$ Retailer Item Price Location.

\subsection{Nested Normal Form (NNF)}

To help achieve our goal, we make use of NNF [19] in this paper. We have proved in [19] that a scheme tree does not permit redundancy with respect to a set of MVDs and FDs if and only if it is in NNF. Thus, our goal in this paper is to extract a largest NNF scheme tree.

Definition 6 A scheme tree $T$ over a set $U$ of attributes is a rooted tree in which every node is a nonempty subset of $U$. Further, the intersection of every pair of nodes in $T$ is empty.

Definition 7 Let $T$ be a scheme tree over a set $U$ of attributes. Let $\operatorname{dom}(A)$ be the set of domain values of an attribute $A$ in $U$. A scheme-tree instance over $T$ is recursively defined as follows:

1. If $T$ has only the root node $A_{1} \cdots A_{n}(n \geq 1)$, a scheme-tree instance over $T$ is a (possibly empty) set of functions $\left\{t_{1}, \ldots, t_{m}\right\}$ such that each $t_{i}(1 \leq i \leq m)$ maps each $A_{j}(1 \leq j \leq n)$ to a value in $\operatorname{dom}\left(A_{j}\right)$.

2. If $T$ has more than one node, then let $T_{1}, \ldots, T_{k}(k \geq 1)$ be the $k$ subtrees of $T$ such that the root node of each $T_{i}$ is a child node of $T$ 's root node. Let $\left\{t_{1}, \ldots, t_{m}\right\}(m \geq 0)$ be the set of functions associated with $T$ 's root node and let $t_{j} \oplus s_{j_{i}}$ mean that the function $t_{j}$ associates with the scheme-tree instance $s_{j_{i}}$ over $T_{i}$ for $t_{j}$. Then, $\cup_{j=1}^{m}\left(\cup_{i=1}^{n} t_{j} \oplus s_{j_{i}}\right)$ is a scheme-tree instance over $T$. 
Although formally defined in Definition 7, scheme-tree instances are most easily understood when visualized and written as are the scheme-tree instances in Figure 2. In Figure 2, we nest attribute names in parentheses in a linear fashion according to their structure and place instance values in buckets (with the outermost bucket omitted).

Let $T$ be a scheme tree. We denote the set of attributes in $T$ by $A \operatorname{set}(T)$. Let $N$ be a node in $T$. Notationally, Ancestor $(N)$ denotes the union of attributes in all ancestors of $N$, including $N$. Similarly, Descendent $(N)$ denotes the union of attributes in all descendants of $N$, including $N$. In a scheme tree $T$, each edge $(V, W)$, where $V$ is the parent of $W$, denotes an MVD Ancestor $(V)$ $\rightarrow$ Descendent $(W)$. Notationally, we use $\operatorname{MVD}(T)$ to denote the set of all MVDs represented by the edges in $T$. By construction, each MVD in $M V D(T)$ is satisfied in the total unnesting of any scheme-tree instance for $T$. Since FDs are also of interest, we use $F D(T)$ to denote the set of FDs that hold in $T$.

Example 4 Figures 2(a), 2(b), and 2(c) show three possible sets of scheme trees and their instances derived from the data in Figure 1(b). As in [19] we use a repeating-group (...)* to denote a nested scheme tree and a bucket to denote a nested scheme-tree instance. Let $T$ be the left scheme tree in Figure 2(c). Each edge in T implies an MVD. Therefore, $M V D(T)$ is equal to \{ Retailer $\rightarrow$ Location, Retailer $\rightarrow$ Item Price Manufacturer, Retailer Item Price $\rightarrow$ Manufacturer $\}$. In addition, $F D(T)$ is equal to $\{$ Retailer Item $\rightarrow$ Price $\}$ as declared in Figure 1(a).

Definition 8 Let $U$ be a set of attributes. Let $M$ be a set of MVDs over $U$ and $F$ be a set of FDs over $U$. Let $T$ be a scheme tree such that $A \operatorname{set}(T) \subseteq U$. T is in NNF with respect to $M \cup F$ if the following conditions are satisfied.

1. Let $D$ be the set of MVDs and FDs that hold for $T$ with respect to $M \cup F$. The set $D$ is equivalent to $M V D(T) \cup F D(T)$ on $\operatorname{Aset}(T)$.

2. For each nontrivial FD $X \rightarrow A$ that holds for $T$ with respect to $M \cup F, X \rightarrow$ Ancestor $\left(N_{A}\right)$ also holds with respect to $M \cup F$, where $N_{A}$ is the node in $T$ that contains $A$.

Example 5 All scheme trees in Figures 2(b) and 2(c) are in NNF. The scheme tree in Figure 2(a), however, is not in NNF. To see this, let $T$ be the scheme tree in Figure 2(a). Then, $M V D(T)$ $=\{$ Retailer $\rightarrow$ Location, Retailer $\rightarrow$ Item Price Manufacturer Factory, Retailer Item Price $\rightarrow$ Manufacturer Factory, Retailer Item Price Manufacturer $\rightarrow$ Factory $\}$, and $F D(T)=\{$ Retailer Item $\rightarrow$ Price $\}$. Now, observe that Manufacturer $\rightarrow$ Factory is a hypergraph-generated MVD that holds in $T$ (obtained by removing Manufacturer from the hypergraph in Figure 1(a)). Using the chase [16], it is easy to show that $\operatorname{MVD}(T) \cup F D(T)$ does not imply Manufacturer $\rightarrow$ Factory and therefore that $T$ violates NNF's Condition 1.

\subsection{Syntactic Covers}

Syntactic covers guarantee that every value and every relationship in an associated instance of a hypergraph can appear in a scheme-tree instance (e.g., that the values and relationships in the 
instance in Figure 1(b) can appear in the scheme tree instances in Figure 2.) Since we are generating storage structures, syntactic coverage is a necessary condition for any set of scheme trees generated for a hypergraph.

In the following, for any subset $S$ of a hypergraph $H$, we use the notation $\bar{S}$ to denote the set $\cup_{E_{i} \in S} E_{i} . \bar{S}$ is simply the set of attributes in some set of hypergraph edges.

Definition 9 A path of a scheme tree $T$ is a sequence of nodes from the root node of $T$ to a leaf node of $T$. Let $H$ be a hypergraph. An attribute $A \in \bar{H}$ appears in a scheme tree $T$ if $A$ is in a node of $T$. A hyperedge $E \in H$ appears in a scheme tree $T$ if there is a path in $T$ whose nodes collectively contain all of $E$ 's attributes. ${ }^{2}$

Definition 10 A scheme tree $T$ syntactically covers a set $S$ of hyperedges if (1) Aset $(T)=\bar{S}$, and (2) every hyperedge in $S$ appears in a path of $T$. A scheme-tree forest $F$ syntactically covers a hypergraph $H$ if there are subsets $S_{1}, \ldots, S_{n}$ of hyperedges in $H$ such that $\overline{S_{1}} \cup \cdots \cup \overline{S_{n}}=\bar{H}$ and there are scheme trees $T_{1}, \ldots, T_{n}$ in $F$ such that $T_{i}$ syntactically covers $S_{i}(1 \leq i \leq n)$.

Example 6 All three sets of scheme trees in Figures 2(a), 2(b) and 2(c) syntactically cover the hypergraph in Figure 1(a). As an example of failure to syntactically cover, consider the first scheme tree in the scheme-tree forest in Figure 2(c). If we remove Price, there is no place for Price values. Clearly, every attribute must appear in the scheme-tree forest. If we remove Manufacturer, although there is still a place for Manufacturer values in the second scheme tree in Figure 2(c), there is no place for the triples that belong to the edge $\{$ Retailer, Item, Price $\}$. Clearly, every edge must appear in a path of some scheme tree.

\section{Extracting a Largest NNF Scheme Tree}

The main algorithm of this paper extracts a largest NNF scheme tree from a reduced acyclic hypergraph and a set $F$ of embedded FDs such that each hyperedge is in BCNF. The algorithm calls several procedures, which are explained in detail in the following sections. As a summary, Step 1 reduces the number of input hyperedges. Step 2 creates a join tree and a set of labels for the acyclic hypergraph. Step 3 constructs a Hasse diagram of a partial order defined on the acyclic hypergraph's labels. Step 4 refines the join tree created in Step 2. Step 5 extracts a largest NNF skeleton from the Hasse diagram. Finally, Step 6 attaches the NNF skeleton's hyperedges to the skeleton to make it a largest NNF scheme tree.

\footnotetext{
${ }^{2}$ Note that the definition of syntactic coverage for this paper differs from the definition in [18]. In [18] the definition requires a hyperedge to appear in contiguous nodes in a path of a scheme tree while the definition here does not. Since we make the universal relation assumption in this paper and we did not for [18], we can relax the condition of syntactic coverage in [18]. For example, consider a reduced, acyclic hypergraph $H=\left\{A V_{1}, A B V_{2}, A B C V_{3}, A C V_{4}\right\}$ and an embedded FD $A C \rightarrow B$. A NNF scheme tree $T$ for $H$ has $A$ as the root node, $A$ 's child nodes are $B$ and $V_{1}, B$ 's child nodes are $C$ and $V_{2}$, and $C$ 's child nodes are $V_{3}$ and $V_{4}$. The hyperedge $A C V_{4}$ does not appear in contiguous nodes in any path in $T$. Nevertheless, $T$ is in NNF and $T$ syntactically covers the entire hypergraph under the definition of syntactic coverage of this paper.
} 


\subsection{The Main Algorithm}

\section{Procedure Main}

Input: a reduced acyclic hypergraph $H$ and a set $F$ of embedded FDs such that each hyperedge in $H$ is in $\mathrm{BCNF}$.

Output: a largest NNF scheme tree.

1. Call Procedure MergeHyperedges.

2. Call Procedure CreateJoinTree.

3. Call Procedure ConstructHasseDiagram0f $\succeq$.

4. Call Procedure MoveLabelsToCenterNodes.

5. Call Procedure ExtractLargestNNFSkeleton.

6. Call Procedure AttachHyperedges.

\subsection{Procedure MergeHyperedges}

Two distinct hyperedges $E_{i}$ and $E_{j}$ are functionally equivalent if $E_{i} \rightarrow E_{j}$ and $E_{j} \rightarrow E_{i}$. Theorem 1 of Section 5.1 states that there is no loss of generality to assume that no two distinct functionally equivalent hyperedges exist. Hence, Procedure MergeHyperedges merges functionally equivalent hyperedges together to reduce the number of input hyperedges. From now on, we can safely assume that no two distinct functionally equivalent hyperedges exist.

\section{Procedure MergeHyperedges}

Input: a reduced acyclic hypergraph $H$ and a set $F$ of embedded FDs such that each hyperedge in $H$ is in $\mathrm{BCNF}$.

Output: a reduced acyclic hypergraph $H$ with no distinct functionally equivalent hyperedges and the same set $F$ of embedded FDs.

1. Call Algorithm 4.4 on page 66 in [16] to compute $E^{+}$for each hyperedge $E \in H$.

2. Put hyperedges $E_{i}$ and $E_{j}$ in the same set if $E_{i}^{+}=E_{j}^{+}$.

3 . For each set $S$ with two or more hyperedges, do:

Merge all hyperedges in $S$ together to form a new hyperedge and add it to $H$.

Remove each hyperedge in $S$ from $H$.

Example 7 Consider the FDs and hyperedges in Figure 4(a). ${ }^{3}$ Every FD is embedded in some hyperedge, every hyperedge is in BCNF, and these hyperedges together constitute an acyclic hypergraph. The hyperedges $A V_{2}$ and $A V_{3}$ in Figure 4(a) are functionally equivalent because $A V_{2}^{+}=A V_{3}^{+}$ $=A V_{2} V_{3}$. Thus, Procedure MergeHyperedges merges $A V_{2}$ and $A V_{3}$ together to form a new hyperedge $A V_{2} V_{3}$ and removes $A V_{2}$ and $A V_{3}$ from $H$. A join tree created by Procedure CreateJoinTree in Section 2.1 for the resulting acyclic hypergraph is shown in Figure 4(b).

\footnotetext{
${ }^{3} V_{1}, \ldots, V_{16}$ are attributes that appear in exactly one hyperedge. Attributes that appear in exactly one hyperedge are not essential for our algorithm.
} 


\begin{tabular}{|c|}
\hline Hyperedges \\
\hline 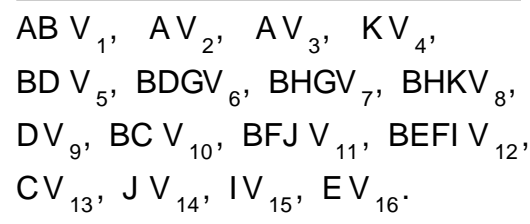 \\
\hline Embedded FDs \\
\hline $\begin{array}{l}A \rightarrow V_{2}, A \rightarrow V_{3}, B \rightarrow A V_{1}, \\
B \rightarrow C V_{10}, C \rightarrow>B V_{10}, \\
I \rightarrow B E F V_{12}, J \rightarrow B F V_{11}, \\
K \rightarrow B H V_{8}\end{array}$ \\
\hline
\end{tabular}

(a)

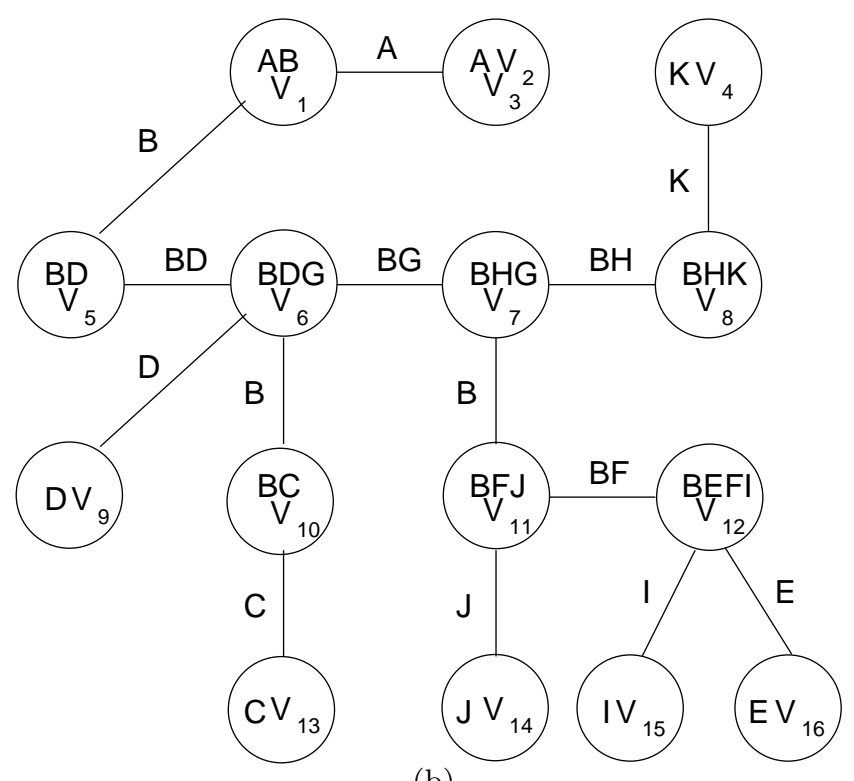

(b)

Figure 4: Merging Functionally Equivalent Hyperedges and Creating a Join Tree.

\subsection{Procedure ConstructHasseDiagramOf $\succeq$}

We now define a partial order on the labels of the input reduced acyclic hypergraph. Later we derive a largest NNF scheme tree from the Hasse diagram of this partial order.

Definition 11 Let $H$ be a reduced acyclic hypergraph and $F$ be a set of embedded FDs. Two distinct labels $L_{i}$ and $L_{j}$ of $H$ are functionally equivalent if $L_{i} \rightarrow L_{j}$ and $L_{j} \rightarrow L_{i}$. Let $C_{1}, \ldots, C_{n}$ be the equivalence classes ${ }^{4}$ of labels of $H$ such that all the labels in each equivalence class $C_{i}$ are pairwise functionally equivalent. We define $\succeq$ to be a partial order on $C_{1}, \ldots, C_{n}$ in which $C_{i} \succeq C_{j}$ if $L_{i} \rightarrow L_{j}$ where $L_{i} \in C_{i}$ and $L_{j} \in C_{j}$.

Lemma 6 of Section 5.3 states that the multiset of labels in any join tree for an acyclic hypergraph is the same. Therefore, the partial order $\succeq$ and its derived Hasse diagram are unique for the input reduced acyclic hypergraph and the embedded FDs.

\section{Procedure ConstructHasseDiagram0f $\succeq$}

Input: a join tree $J$ and a set $F$ of embedded FDs such that each node in $J$ is in BCNF.

Output: the Hasse diagram of $\succeq$.

1. Call Algorithm 4.4 on page 66 in [16] to compute $L^{+}$for each label $L$ of $J$.

2. Put labels $L_{i}$ and $L_{j}$ in the same equivalence class if $L_{i}^{+}=L_{j}^{+}$.

3. For two equivalence classes $C_{i}$ and $C_{j}, C_{i} \succeq C_{j}$ if $L_{i}^{+} \supseteq L_{j}^{+}$where $L_{i} \in C_{i}$ and

\footnotetext{
${ }^{4}$ An equivalence class is a set, not a multiset.
} 


\section{$L_{j} \in C_{j}$.}

4. Generate the Hasse diagram of $\succeq$.

Example 8 The labels $B$ and $C$ in Figure 4(b) are in the same equivalence class because they are functionally equivalent. On the other hand, each of the other labels in Figure 4(b) is in a different equivalence class. The Hasse diagram of $\succeq$ is shown in Figure $5(\mathrm{a})$, in which $\{B D\} \succeq\{B, C\}$, $\{B D\} \succeq\{D\}$, and $\{B, C\} \succeq\{A\}$, and so on.

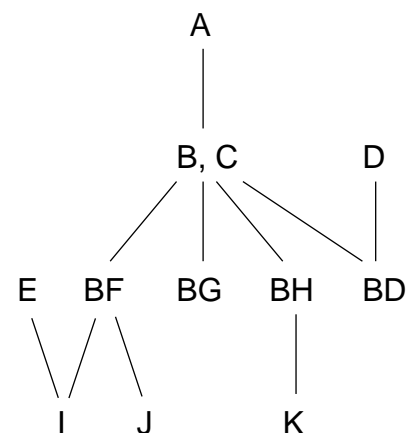

(a)

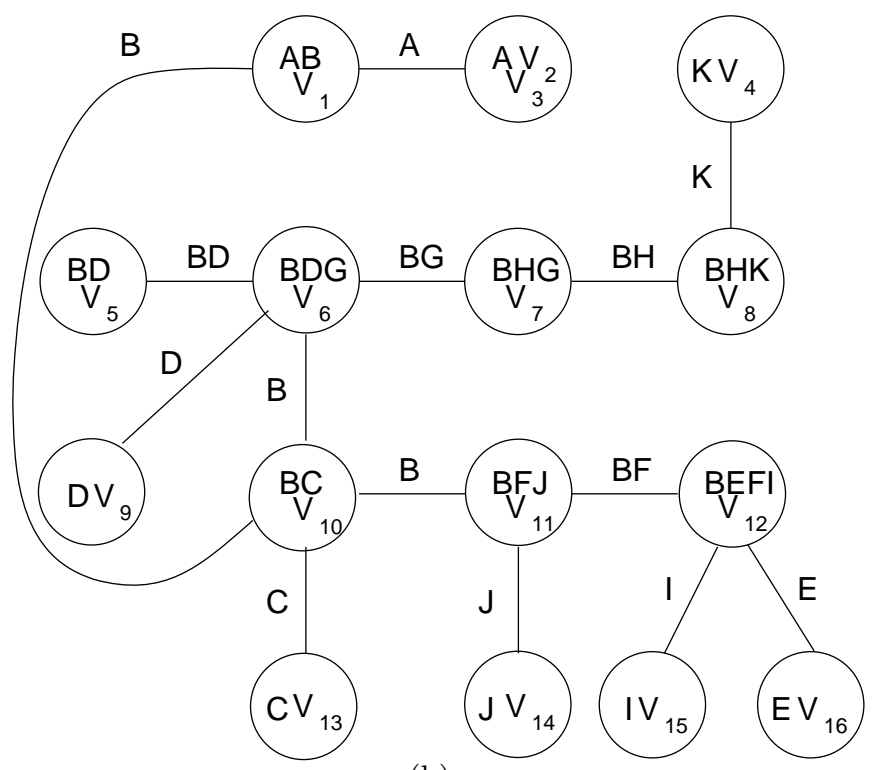

(b)

Figure 5: Constructing the Hasse diagram of $\succeq$ and Moving Labels to Center Nodes.

\subsection{Procedure MoveLabelsToCenterNodes}

Lemmas 7 and 8 of Section 5.3 together state that all distinct labels of any equivalence class of labels are incident with a unique common node in a join tree. We call such a node the center node of the equivalence class. Procedure MoveLabelsToCenterNodes makes all labels in a join tree that appear in an equivalence class incident with the equivalence class's center node.

\section{Procedure MoveLabelsToCenterNodes}

Input: a join tree $J$ and a set of equivalence classes of labels in $J$.

Output: a modified join tree $J$ with all labels in $J$ that appear in an equivalence class of labels incident with the equivalence class's center node.

1. For each equivalence class $C$ with two or more distinct labels, do:

Locate the center node $E$ of $C$.

For each edge $\left\{E_{i}, E_{j}\right\}$ in $J$ such that $E_{i} \cap E_{j}$ is a label in $C$, do:

Remove $\left\{E_{i}, E_{j}\right\}$ from $J$. 


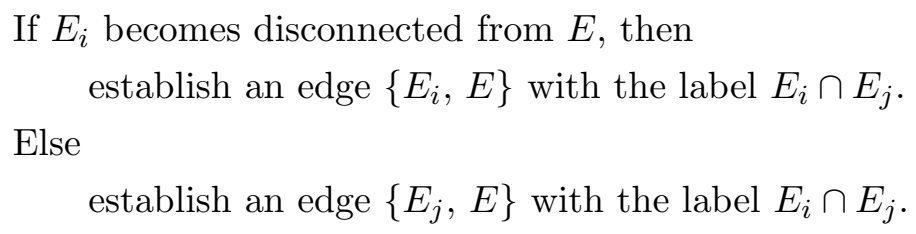

If $E_{i}$ becomes disconnected from $E$, then

establish an edge $\left\{E_{i}, E\right\}$ with the label $E_{i} \cap E_{j}$.

Else

establish an edge $\left\{E_{j}, E\right\}$ with the label $E_{i} \cap E_{j}$.

2. For each equivalence class $C$ with exactly one label, do:

Arbitrarily choose one node of an edge in $J$ with that label.

Designate that node as the center node for $C$.

Repeat the inner for-loop in Step 1.

Example 9 Since $B \rightarrow C$ and $C \rightarrow B$, we have the equivalence class of labels $\{B, C\}$. The center node for $\{B, C\}$ is $B C V_{10}$ in Figure 4(b). The result of applying Procedure MoveLabelsToCenterNodes on the join tree in Figure 4(b) is shown in Figure 5(b).

\subsection{Procedure ExtractLargestNNFSkeleton}

Theorem 2 of Section 5.2 states that if an NNF scheme tree syntactically covers some hyperedges, the hyperedges must be the nodes in a connected subtree of a join tree. Additionally, Theorem 3 of Section 5.3 states that to satisfy NNF, this connected subtree cannot have any critical node. Based on these two theorems, creating a largest NNF scheme tree that contains the greatest number of hyperedges is the same as creating a largest NNF scheme tree that syntactically covers the nodes in a connected subtree of a join tree where (1) the number of nodes in the connected subtree is the greatest and (2) the connected subtree has no critical nodes. To accomplish this goal, we first find a largest NNF skeleton in the Hasse diagram of $\succeq$ that contains the greatest number of labels. Then, we attach the hyperedges with which these labels are incident to this skeleton to make it a largest NNF scheme tree. The definitions of these concepts now follow.

Definition 12 A connected subtree of a join tree $T$ is inductively defined as follows: (1) A single node in $T$ is a connected subtree of $T$. (2) If $N^{\prime}$ is a node in a connected subtree $T^{\prime}$ of $T$ and $N$ is a node in $T$ such that $\left\{N, N^{\prime}\right\}$ is an edge in $T$, then $T^{\prime}$ augmented with the node $N$ and the edge $\left\{N, N^{\prime}\right\}$ is a connected subtree of $T$. Let $T^{\prime}$ be a connected subtree of a join tree. The notation $\overline{T^{\prime}}$ denotes the union of all the hyperedges that are nodes in $T^{\prime}$.

Definition 13 Let $H$ be an acyclic hypergraph and $F$ be a set of embedded FDs in $H$. Let $J$ be a join tree for $H$ and $S$ be a connected subtree of $J$, which is not necessarily a proper subset. A label $L$ of $H$ belongs to $S$ if there is an edge $E$ in $S$ such that $E$ 's label is $L$. A node $N$ in $S$ is critical with respect to $S$ if there are two labels $L_{i}$ and $L_{j}$ belonging to $S$ such that $L_{i} \nrightarrow L_{j}, L_{j} \nrightarrow L_{i}$, and $\left(L_{i} \cup L_{j}\right) \subseteq N$. If $S$ is actually $J$, then we may simply call a node of $J$ critical without having to make any reference to $S$.

Definition 14 Given an equivalence class $C$ in the Hasse diagram of $\succeq$, any tree rooted at $C$ extracted from the Hasse diagram of $\succeq$ is called a skeleton. Let $K$ be a skeleton and $J$ be a join 
tree. $K$ 's induced set of edges is the set $\{E$ is an edge in $J: E$ 's label appears in an equivalence class in $K\}$. A NNF skeleton is a skeleton whose induced set of edges constitutes a connected subtree of $J$ and the connected subtree has no critical nodes.

Definition 15 Let $C_{i}$ and $C_{k}$ be two equivalence classes of labels such that $C_{i}$ is a parent node of $C_{k}$ in the Hasse diagram of $\succeq$. $C_{k}$ is a nontrivial child of $C_{i}$, or $C_{k} \succeq C_{i}$ nontrivially, if for each $L_{i} \in C_{i}$ and for each $L_{k} \in C_{k}, L_{i} \not L_{k}$. On the other hand, if there are labels $L_{i} \in C_{i}$ and $L_{k} \in C_{k}$ such that $L_{i} \subset L_{k}$, then $C_{k}$ is a trivial child of $C_{i}$, or $C_{k} \succeq C_{i}$ trivially.

Theorem 4 of Section 5.4 proves that Procedure ExtractLargestNNFSkeleton indeed outputs NNF skeletons.

\section{Procedure ExtractLargestNNFSkeleton}

Input: the Hasse diagram of $\succeq$ and the modified join tree $J$.

Output: a largest NNF skeleton.

1. For each equivalence class $C$ of labels in the Hasse diagram of $\succeq$, do:

Associate with $C$ an integer variable labelCnt and set $C$.labelCnt $=0$.

Associate with $C$ a set of edges in $J$ called myEdges where C.myEdges = $\{E$ is an edge in $J: E$ 's label appears in $C\}$.

2. For each root node $R$ in the Hasse diagram of $\succeq$, do:

Call Procedure CalculateLabelCnt $(R)$.

3. Select a root node $R$ in the Hasse diagram of $\succeq$ with the greatest labelCnt.

4. Return the NNF skeleton rooted at $R$.

Procedure CalculateLabel $\operatorname{Cnt}(C$ : an equivalence class in the Hasse diagram of $\succeq)$

1. If C.labelCnt $=0$, then

For each nontrivial child $D$ of $C$, do:

Call Procedure CalculateLabelCnt $(D)$.

C.labelCnt $=$ C.labelCnt + D.labelCnt .

For each trivial child $D$ of $C$, do:

Call Procedure CalculateLabelCnt $(D)$.

While there is an unmarked trivial child of $C$, do:

Set $\max D$ to an unmarked trivial child of $C$ with the greatest labelCnt.

Mark $\max D$.

For each other unmarked trivial child $D$ of $C$, do:

If the path between $D$ 's center node and maxD's center node

in $J$ does not contain any edge in C.myEdges, then

Remove $D$ as a trivial child of $C$.

For each marked trivial child $D$ of $C$, do:

C.labelCnt $=$ C.labelCnt + D.labelCnt.

C.labelCnt $=$ C.labelCnt + the size of C.myEdges. 
Example 10 Steps 1, 3, and 4 of Procedure ExtractLargestNNFSkeleton are straightforward. Let us focus on Step 2. With respect to the Hasse diagram in Figure 5(a), there are three root nodes, namely $\{E\},\{A\}$, and $\{D\}$. Suppose the root node $R$ in Step 2 is $\{A\}$. Initially, $\{A\}$.labelCnt $=0$. Thus, Procedure CalculateLabelCnt enters the if-statement. Then, Procedure CalculateLabelCnt recursively calls itself until it reaches the leaf nodes of the Hasse diagram. The five leaf nodes in the Hasse diagram are $\{I\},\{J\},\{B G\},\{K\}$, and $\{B D\}$. Since none of these equivalence classes of labels has a child and since there is only one label in Figure 5(b) that appears in each of these equivalence classes, $\{I\}$.labelCnt $=1,\{J\}$.labelCnt $=1,\{B G\}$.labelCnt $=$ $1,\{K\}$.labelCnt $=1$, and $\{B D\}$.labelCnt $=1$. As Procedure CalculateLabelCnt unwinds from recursion, since $\{I\}$ and $\{J\}$ are nontrivial children of $\{B F\},\{I\}$.labelCnt and $\{J\}$.labelCnt are added to $\{B F\}$.labelCnt. And because there is only one label in Figure $5(\mathrm{~b})$ that appears in $\{B F\}$, $\{B F\}$.labelCnt $=3$, as Figure 6(a) shows. The same reasoning applies to $\{B H\}$.labelCnt. Now, Procedure CalculateLabelCnt unwinds to the equivalence class $\{B, C\}$ in Figure $5(\mathrm{a}) .\{B F\}$ is selected first because $\{B F\}$.labelCnt is the greatest among all $\{B, C\}$ 's trivial children. However, there is a label $B$ in between of $\{B F\}$ 's center node and the center node of the other trivial child of $\{B, C\}$ in Figure 5(b). Thus, the inner for-loop of the while-loop in Procedure CalculateLabelCnt has no effect. The next trivial child to be selected is $\{B H\}$. However, with respect to Figure $5(\mathrm{~b})$, neither the path between $\{B H\}$ 's center node and $\{B G\}$ 's center node nor the path between $\{B H\}$ 's center node and $\{B D\}$ 's center node contains any label $B$ or label $C$. Thus, Procedure CalculateLabelCnt removes $\{B G\}$ and $\{B D\}$ as trivial children of $\{B, C\}$. There are four labels in Figure 5(b) that appear in $\{B, C\}$. Thus, $\{B, C\}$.labelCnt $=\{B F\}$.labelCnt + $\{B H\}$.labelCnt $+4=3+2+4=9$. Finally, Procedure CalculateLabelCnt unwinds back to $\{A\}$ and there is only one label in Figure 5(b) that appears in $\{A\}$. Thus, $\{A\}$.labelCnt $=\{B$, $C\}$.labelCnt $+1=9+1=10$, as Figure 6(b) shows. $\{E\}$.labelCnt and $\{D\}$.labelCnt are calculated similarly.

\subsection{Procedure AttachHyperedges}

The last step of our algorithm attaches hyperedges to a largest NNF skeleton.

\section{Procedure AttachHyperedges}

Input: a largest NNF skeleton $T$ and the modified join tree $J$.

Output: a largest NNF scheme tree.

1. Let $S$ be $T$ 's induced set of edges in $J$.

2. For each node $E$ in $S$, do:

Find the lowest node $N$ in $T$ such that $N$ contains a label $L$ where $L \subseteq E$.

Let $N_{E}=\{A \in E: A$ does not appear in any label in any equivalence class of $T\}$.

If $N_{E} \neq \emptyset$, then

Add $N_{E}$ as a child node to $N$ in $T$.

3. For each node $N$ in $T$, do: 


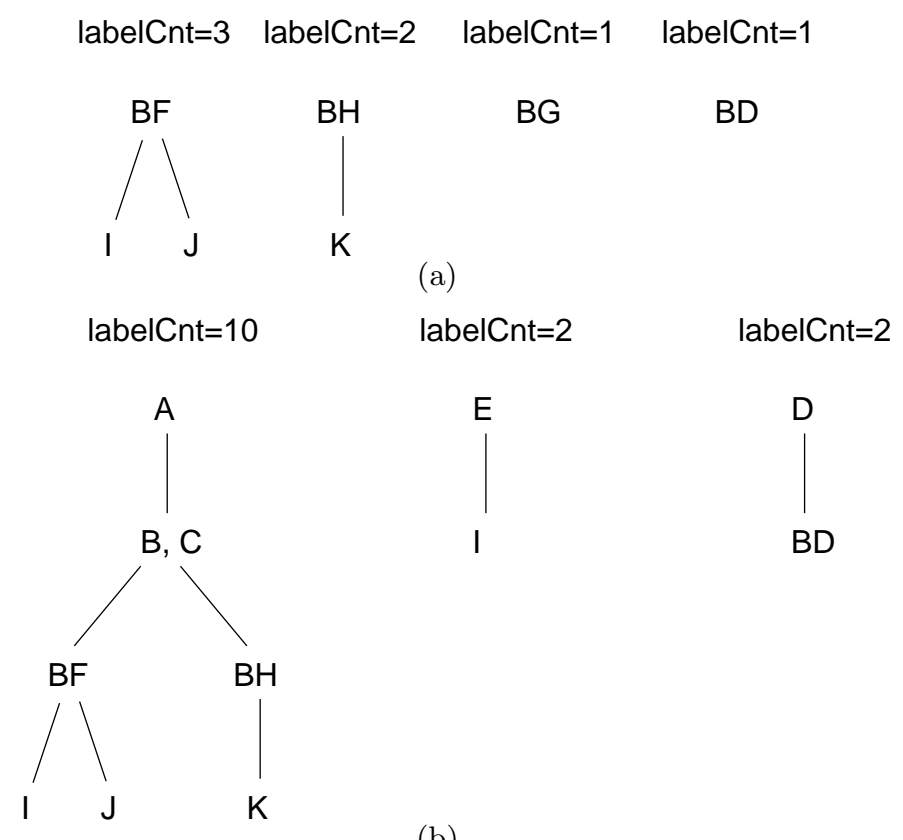

Figure 6: Calculating labelCnt for each Equivalence Class in the Hasse diagram of $\succeq$.

Merge all labels in $N$ together.

4. For each child node $N$ in $T$, do:

If an attribute $A \in N$ is also in $N$ 's parent node, then

Remove $A$ from $N$.

Example 11 Figure 7(a) shows how Procedure AttachHyperedges turns an NNF skeleton into an NNF scheme tree. As an example, the lowest node $N$ is $\{B, C\}$ for the hyperedge $A B V_{1}$. Thus, $V_{1}$ is added as a child node to $\{B, C\}$. Then, Procedure AttachHyperedges merges all labels together in every node and removes every redundant attribute. Figure 7(b) shows the connected subtree defined by the set $S$ from which the NNF scheme tree in Figure 7(a) is constructed.

\section{Experimental Evaluation}

As Theorem 4 of Section 5.4 asserts, the algorithms that underlie Procedure MergeHyperedges and Procedure CreateJoinTree have been well-studied in the literature and have been proved to run in time polynomial in the size of the input. In addition, Theorem 4 also shows that Procedure AttachHyperedges runs in time linear with respect to the size of the input. Thus, in our experiments, we focus on Procedure ConstructHasseDiagram0f $\succeq$, Procedure MoveLabelsToCenterNodes, and Procedure ExtractLargestNNFSkeleton. We have implemented these procedures in a Visual Basic 2008 program, which first randomly generates join trees and equivalence classes of labels and then extracts largest NNF skeletons from them. The computer used in our experiments is a Dell 

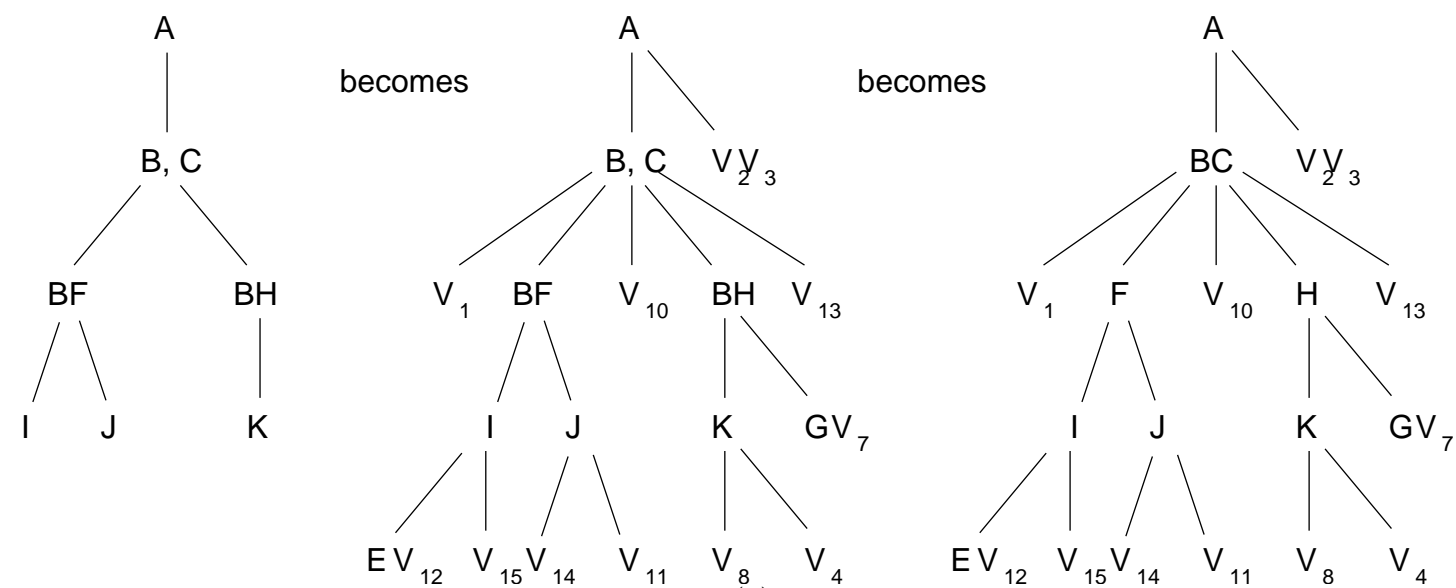

(a)

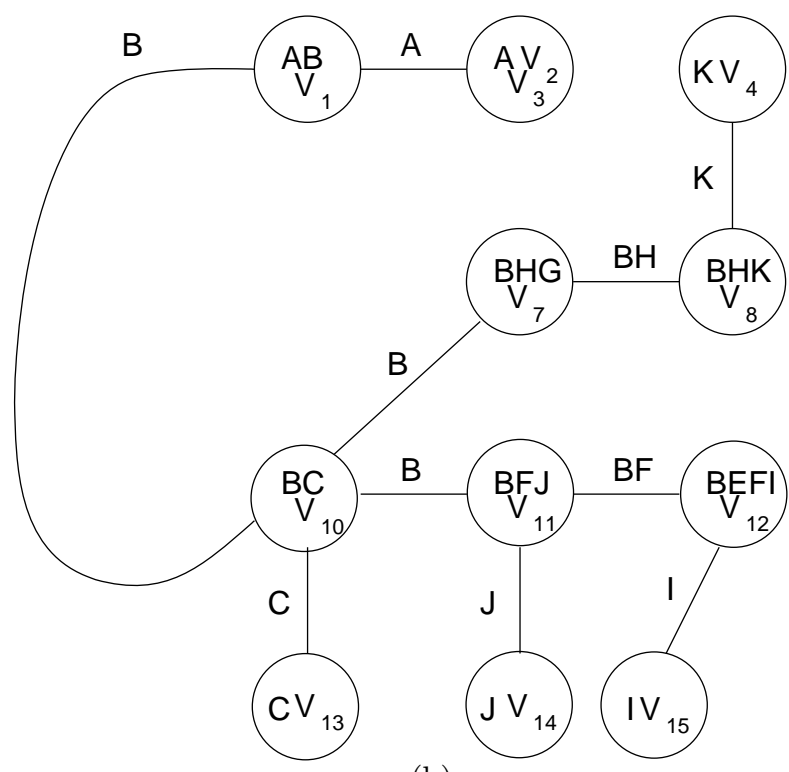

(b)

Figure 7: Turning an NNF Skeleton to an NNF Scheme Tree.

desktop PC with an E6300 Intel Core $2 \mathrm{CPU}$ running at 1.86 and $1.87 \mathrm{GHz}$ with $2045 \mathrm{MBs}$ of memory. The operating system is Windows Vista Business Edition.

Figure 8 shows the time taken by the simulation program, measured in milliseconds (ms), when there are 2500, 5000, 7500, and 10000 hyperedges. Although the definition of a join tree does not require a root node, having a root node in a join tree makes our implementation much easier. As a result, the terms "parent nodes" and "child nodes" are applicable to our join trees. In our experiments, each internal node of a join tree randomly has 1 or any number up to maxFanout child nodes, where maxFanout is a variable that is set to 1, 3, 5, 10, 15, 20, 25, 50, 100 or 200. By increasing the value of maxFanout, more labels are clustered into an equivalence class of labels. This in turn reduces their number. Fewer equivalence classes of labels results in fewer comparisons needed to construct the partial order $\succeq$. Thus, the program takes less time to complete. 
However, Figure 8 also shows that the time needed to complete the program levels off as the value of maxFanout increases. One of the reasons for this phenomenon is that overhead operations take more time as the value of maxFanout increases, which cancels out the advantage of increasing maxFanout.

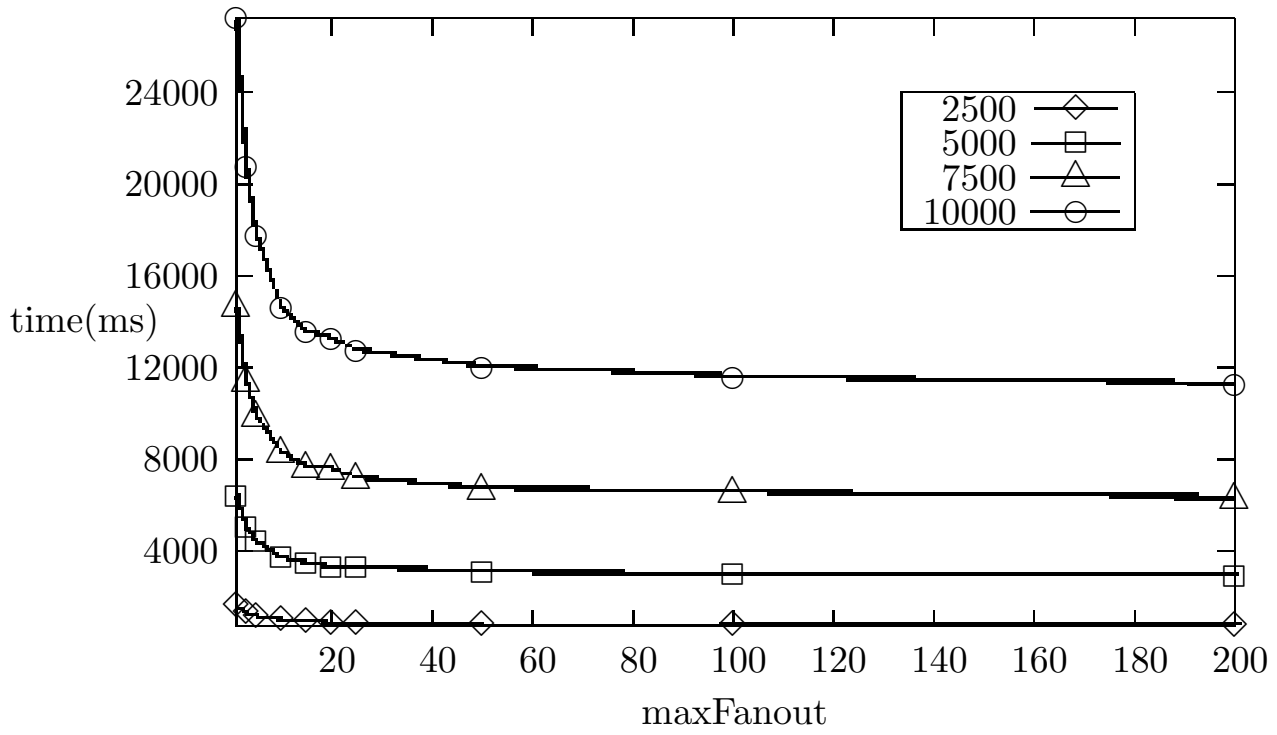

Figure 8: Plotting time against maxFanout.

Another observation about Figure 8 is that for each of the 10 values of maxFanout, doubling the number of hyperedges quadruples the time needed to find a largest NNF skeleton. This gives a hint that these three procedures as a whole run in time polynomial in the size of the input.

Figure 9 provides further evidence for this claim. For each of the 10 values of maxFanout, we plot $n^{2} /$ time against $n$, where $n$ is the number of hyperedges. In all four values of $n$, the ratio between $n^{2}$ and time (i.e., $n^{2} /$ time) is relatively stable for a fixed value of maxFanout. Since our join trees and equivalence classes of labels are randomly generated, the results in Figure 9 suggest that given that all other conditions remain the same, on average Procedure ConstructHasseDiagram0f $\succeq$, Procedure MoveLabelsToCenterNodes, and Procedure ExtractLargestNNFSkeleton considered as a whole run in quadratic time in the size of the input.

\section{Proofs for Claims}

\subsection{Acyclic Hypergraphs and Functionally Equivalent Hyperedges}

Theorem 1, the main result of this section, states that there is no loss of generality to assume that no two distinct functionally equivalent hyperedges exist. However, before we can prove Theorem 1, we need to prove several lemmas. 


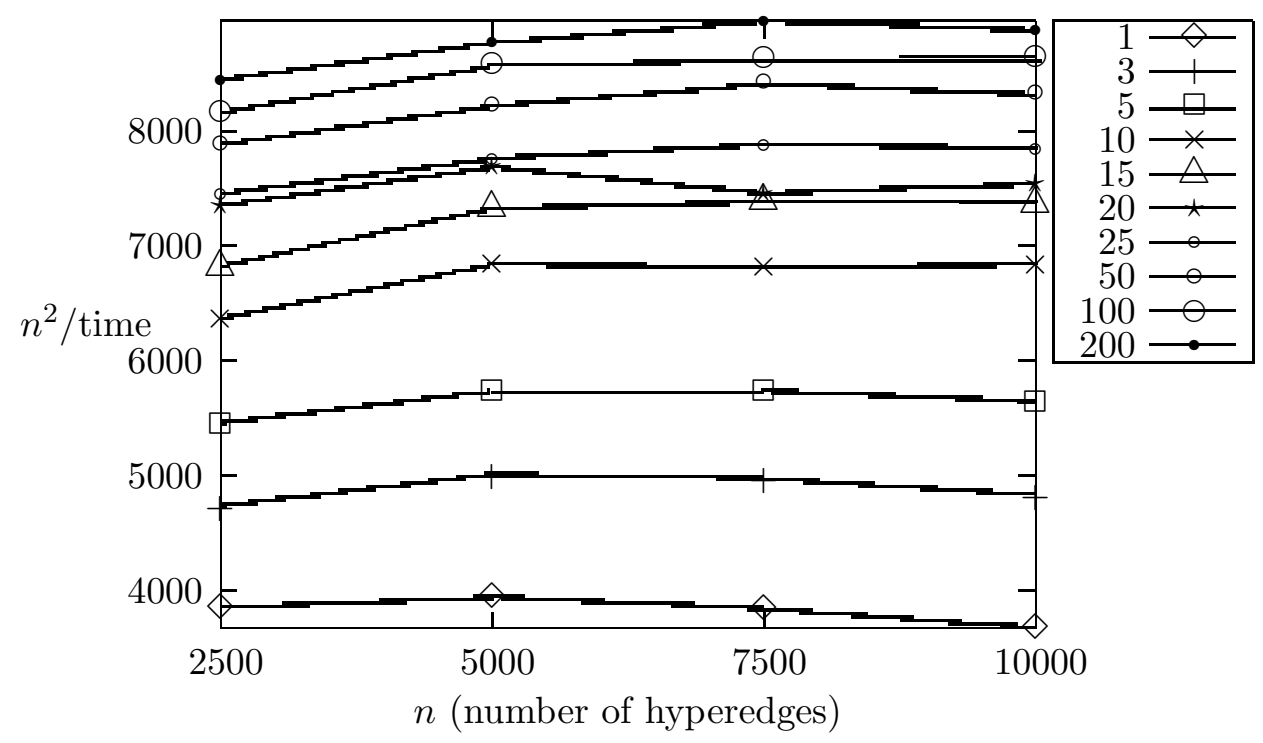

Figure 9: Plotting $n^{2} /$ time against $n$ (number of hyperedges).

Lemma 1 In a join tree $T$ for a reduced, acyclic hypergraph, for any two distinct hyperedges $E_{i}$ and $E_{j}$ and for every attribute $A$ in $E_{i} \cap E_{j}$, the label of each edge along the unique path between $E_{i}$ and $E_{j}$ in $T$ contains $A$.

Proof. See [3].

Let $J$ be a join tree for an acyclic hypergraph $H$ and $\left\{E_{i}, E_{j}\right\}$ be an edge in $J$. We use $J_{i}$ to denote the connected subtree of $J$ that contains the node $E_{i}$ if the edge $\left\{E_{i}, E_{j}\right\}$ were removed from $J$. Likewise, $J_{j}$ denotes the connected subtree of $J$ that contains the node $E_{j}$ if the edge $\left\{E_{i}, E_{j}\right\}$ were removed from $J$. To demonstrate how to obtain $J_{i}$ and $J_{j}$ from $J$, we may imagine cutting along the curved dashed lines in Figure 10. Let $F$ be a set of embedded FDs in $H$. An FD $X \rightarrow Y \in F$ is inside of $J_{i}$ if $X Y \subseteq \bar{J}_{i} ;{ }^{5}$ otherwise, $X \rightarrow Y$ is outside of $J_{i}$. Note that it is possible for an FD to be inside of both $J_{i}$ and $J_{j}$ because $\bar{J}_{i}$ and $\bar{J}_{j}$ are not disjoint. Let $W^{+}$be the closure of a set $W$ of attributes. In the following, we say an FD $X \rightarrow Y \in F$ is used in the derivation of $W^{+}$if $X \rightarrow Y$ is used in the second step of this process: (1) $W^{+}:=W$ initially; (2) $W^{+}:=W^{+} \cup Y$ if $X \subseteq W^{+}$and $Y-W^{+} \neq \emptyset$.

Lemma 2 Let $J$ be a join tree for a reduced, acyclic hypergraph $H$ and $\left\{E_{i}, E_{j}\right\}$ be an edge in $J$. Let $F$ be a set of embedded FDs in $H$. For any set $W$ of attributes such that $W \subseteq \bar{J}_{i}$, if $X \rightarrow Y \in F$ is an FD that is outside of $J_{i}$ and is used in the derivation of $W^{+}$, then there is a subset $E_{i}^{\prime}$ of $E_{i}$ such that $E_{i}^{\prime} \rightarrow Y$.

Proof. Let $X_{1} \rightarrow Y_{1} \in F$ be the first FD that is outside of $J_{i}$ and is used in the derivation of $W^{+}$. Since the FDs used before $X_{1} \rightarrow Y_{1}$ for generating $W^{+}$are all inside of $J_{i}, X_{1} \subseteq \bar{J}_{i}$. Since $X_{1} \rightarrow Y_{1}$

\footnotetext{
${ }^{5}$ Recall that the notation $\bar{J}_{i}$ denotes the union of all the hyperedges that are nodes in $J_{i}$.
} 


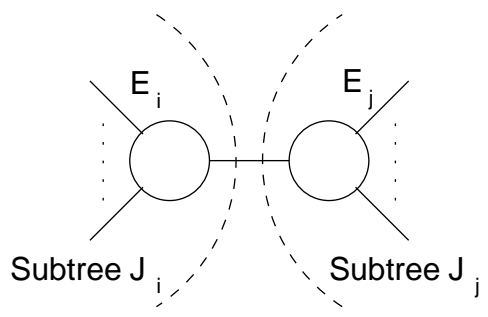

Figure 10: The Connected Subtrees $J_{i}$ and $J_{j}$ of Lemma 2.

is outside of $J_{i}$ and $X_{1} \subseteq \bar{J}_{i}$, by Lemma 1, it must be that $X_{1} \subseteq E_{i}$. Thus, the basis is established. Assume the lemma is true for $k(k \geq 1)$ or less FDs in $F$ that are outside of $J_{i}$ and are used in the derivation of $W^{+}$. Now, consider another FD $X_{k+1} \rightarrow Y_{k+1} \in F$ that is outside of $J_{i}$ and is used in the derivation of $W^{+}$. We first partition $X_{k+1}$ into two sets: $X_{k+1} \cap \bar{J}_{i}$ and $X_{k+1}-\bar{J}_{i}$. Since $X_{k+1} \rightarrow Y_{k+1}$ is outside of $J_{i}$, by Lemma $1, X_{k+1} \cap \bar{J}_{i}$ is a subset of $E_{i}$. Now, consider an attribute $A$ in $X_{k+1}-\bar{J}_{i}$. Since $A \in X_{k+1}$ and $X_{k+1} \rightarrow Y_{k+1}$ is used in generating $W^{+}, A \in W^{+}$. Then, before applying $X_{k+1} \rightarrow Y_{k+1}$ it must be that $A$ has been added to $W^{+}$by an FD in $F$ that is outside of $J_{i}$. By the induction hypothesis, there is a subset $E_{A}^{\prime}$ of $E_{i}$ such that $E_{A}^{\prime} \rightarrow A$. Hence, by forming the union of every $E_{A}^{\prime}$ for each $A$ in $X_{k+1}-\bar{J}_{i}$ and $X_{k+1} \cap \bar{J}_{i}$, there is a subset $E_{i}^{\prime}$ of $E_{i}$ such that $E_{i}^{\prime} \rightarrow Y_{k+1}$.

Example 12 Consider the edge with the label $B G$ in Figure 4(b). On the different sides of this edge are the attribute $J$ and the FD $B \rightarrow A V_{1}$. The attribute $A$ is added to $J^{+}$by $B \rightarrow A V_{1}$; and thus by Lemma 2 , the node $B H G V_{7}$ has a subset, namely $B$, that functionally determines $A$ (i.e., $B \rightarrow A)$.

Similar to what we have done for Lemma 2, we define some terms for Lemma 3 . Let $J$ be a join tree for an acyclic hypergraph $H$ and $J^{\prime}$ be a connected subtree of $J$. Let $F$ be a set of embedded FDs in $H$. An FD $X \rightarrow Y \in F$ is inside of $J^{\prime}$ if $X Y \subseteq \overline{J^{\prime}}$; otherwise, $X \rightarrow Y$ is outside of $J^{\prime}$. Notationally, we let $F^{+}$be the closure of $F, F^{+}[E]$ be the set $\left\{X \rightarrow Y \in F^{+}: E \in H\right.$ and $X Y \subseteq E\}$ and $F^{+}\left[J^{\prime}\right]$ be the set $\cup_{E \in S} F^{+}[E]$ where $S$ is the set $\left\{E \in H: E\right.$ is a node in $\left.J^{\prime}\right\}$.

Lemma 3 Let $J$ be a join tree for a reduced, acyclic hypergraph $H$ and $J^{\prime}$ be a connected subtree of $J$. Let $F$ be a set of embedded FDs in $H$. For any set $W$ of attributes such that $W \subseteq \overline{J^{\prime}}, F^{+}\left[J^{\prime}\right]$ is sufficient to derive $W^{+} \cap \overline{J^{\prime}}$.

Proof. Since $J^{\prime}$ is a connected subtree of $J$, removing the nodes (hyperedges) in $J^{\prime}$ from $J$ will partition the remaining nodes in $J$ into one or more connected subtrees $J_{1}, J_{2}, \ldots, J_{p}(p \geq 1)$. Two of them are shown in Figure 11, in which the dashed lines outline the boundaries of $J_{i}, J_{j}$ and $J^{\prime}$. In addition, for each $J_{i}(1 \leq i \leq p)$, there is a node called $E_{i}$ in $J^{\prime}$ that connects directly to a node in $J_{i}$.

If we only need the FDs in $F$ that are inside of $J^{\prime}$ to derive $W^{+} \cap \overline{J^{\prime}}$, this lemma is vacuously true. Hence, suppose we need some FDs in $F$ that are outside of $J^{\prime}$ to generate $W^{+} \cap \overline{J^{\prime}}$. We 


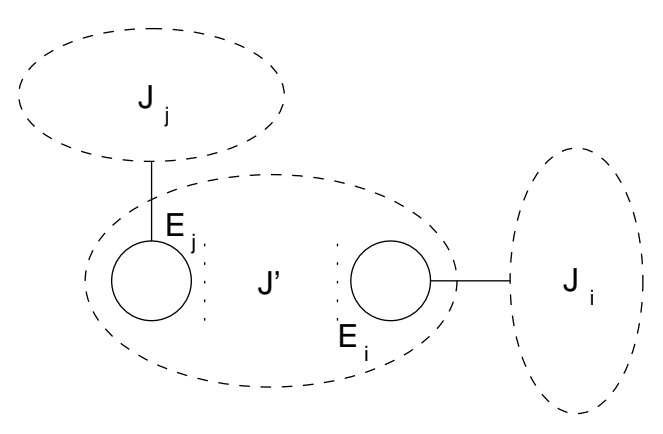

Figure 11: The Connected Subtrees $J^{\prime}, J_{i}$ and $J_{j}$ of Lemma 3.

now describe a procedure that derives $W^{+} \cap \overline{J^{\prime}}$ by using the FDs in $F^{+}\left[J^{\prime}\right]$. Thus, we demonstrate that $F^{+}\left[J^{\prime}\right]$ is sufficient to derive $W^{+} \cap \overline{J^{\prime}}$. Without loss of generality, we assume the right-hand side of each FD in $F$ is a single attribute. For this procedure, we declare $F^{\prime}$, a set of FDs, that is continually a subset of $F^{+}\left[J^{\prime}\right] . F^{\prime}$ is initially set to $\left\{X \rightarrow A \in F: X \rightarrow A\right.$ is inside of $\left.J^{\prime}\right\}$. We then apply the FDs in $F^{\prime}$ to generate $W^{+}$until no more attribute can be added. Assume $n(n \geq 0)$ such FDs are applied in this order:

$$
\begin{gathered}
X_{1} \rightarrow A_{1} \in F^{\prime}, \\
X_{2} \rightarrow A_{2} \in F^{\prime}, \\
\vdots \\
X_{n} \rightarrow A_{n} \in F^{\prime} .
\end{gathered}
$$

Because these $n$ FDs are all in $F^{\prime}, A_{1} \in \overline{J^{\prime}}, A_{2} \in \overline{J^{\prime}}, \ldots$, and $A_{n} \in \overline{J^{\prime}}$. At this point, we have to apply some FDs in $F-F^{\prime}$ in order to continue to add attributes. Assume $m(m \geq 2)$ such FDs are applied in this order:

$$
\begin{gathered}
X_{n+1} \rightarrow A_{n+1} \in F-F^{\prime}, \\
\vdots \\
X_{n+m-1} \rightarrow A_{n+m-1} \in F-F^{\prime}, \\
X_{n+m} \rightarrow A_{n+m} \in F-F^{\prime} .
\end{gathered}
$$

Without loss of generality, we assume that these $m$ FDs are selected in such a way that $A_{n+1}, \ldots$, $A_{n+m-1}$ must all be added to $W^{+}$before $A_{n+m}$ can be added to $W^{+}$, and also $A_{n+1} \notin \overline{J^{\prime}}, \ldots$, $A_{n+m-1} \notin \overline{J^{\prime}}$, and $A_{n+m} \in \overline{J^{\prime}}$. Since $\left(\overline{J_{i}}-\overline{J^{\prime}}\right) \cap\left(\overline{J_{j}}-\overline{J^{\prime}}\right)=\emptyset$ when $i \neq j(1 \leq i, j \leq p)$, our assumption implies that the FDs $X_{n+1} \rightarrow A_{n+1}, \ldots, X_{n+m-1} \rightarrow A_{n+m-1}, X_{n+m} \rightarrow A_{n+m}$ are all inside of the same connected subtree $J_{i}$. Note that since $A_{n+m} \in \overline{J^{\prime}}$ and $X_{n+m} \rightarrow A_{n+m}$ is outside of $J^{\prime}$, by Lemma $1, A_{n+m} \in E_{i}$.

By Lemma 2, for each $X_{j} \rightarrow A_{j}(n+1 \leq j \leq n+m)$, there is a subset $E_{i_{j}}$ of $E_{i}$ such that $E_{i_{j}} \rightarrow A_{j}$. We now show by induction that $F^{\prime}$ implies $W \rightarrow E_{i_{j}}(n+1 \leq j \leq n+m)$. For the FD $X_{n+1} \rightarrow A_{n+1}$, since $X_{n+1} \rightarrow A_{n+1}$ is outside of $J^{\prime}$ and $X_{n+1} \subseteq W A_{1} \cdots A_{n} \subseteq \overline{J^{\prime}}$, by Lemma 1 , $X_{n+1} \subseteq E_{i}$. Thus, $X_{n+1}$ is the subset of $E_{i}$ that we want; and obviously $F^{\prime}$ implies $W \rightarrow X_{n+1}$. 
Hence, the basis is established. Now, consider an FD $X_{k} \rightarrow A_{k}$ for some $k$ where $n+1<k \leq n+m$. With respect to the order of applying the FDs, $X_{k} \subseteq W A_{1} \cdots A_{n} A_{n+1} \cdots A_{k-1}$. We now partition $X_{k}$ into two sets: $X_{k} \cap \overline{J^{\prime}}$ and $X_{k}-\overline{J^{\prime}}$. Since $A_{n+1}, A_{n+2}, \ldots, A_{k-1}$ are not in $\overline{J^{\prime}}$, therefore the argument for $X_{k} \cap \overline{J^{\prime}}$ is the same as that for $X_{n+1}$. That is, $X_{k} \cap \overline{J^{\prime}} \subseteq W A_{1} \cdots A_{n} \subseteq \overline{J^{\prime}}$, $X_{k} \cap \overline{J^{\prime}} \subseteq E_{i}$ and $F^{\prime}$ implies $W \rightarrow X_{k} \cap \overline{J^{\prime}}$. Now, consider an attribute $A \in X_{k}-\overline{J^{\prime}}$. Since $A \in X_{k}$ and $A \notin \overline{J^{\prime}}, A$ must be added to $W^{+}$by an FD before $X_{k} \rightarrow A_{k}$ in the above order. By Lemma 2, there is a subset $E_{A}$ of $E_{i}$ such that $E_{A} \rightarrow A$; and by the induction hypothesis, $F^{\prime}$ implies $W \rightarrow E_{A}$. Thus, if we let $S$ be the union of every $E_{A}$ for each attribute $A \in X_{k}-\overline{J^{\prime}}$, then $S \cup\left(X_{k} \cap \overline{J^{\prime}}\right)$ is a subset of $E_{i}, F^{\prime}$ implies $W \rightarrow S \cup\left(X_{k} \cap \overline{J^{\prime}}\right), S \cup\left(X_{k} \cap \overline{J^{\prime}}\right) \rightarrow X_{k}$ and $S \cup\left(X_{k} \cap \overline{J^{\prime}}\right) \rightarrow Y_{k}$. This means $S \cup\left(X_{k} \cap \overline{J^{\prime}}\right)$ is the subset of $E_{i}$ that we want ${ }^{6}$ and our induction step is complete. Now, by setting $k=n+m$, we have $F^{\prime}$ implies $W \rightarrow E_{i_{n+m}}$ where $E_{i_{n+m}} \subseteq E_{i}$ and $E_{i_{n+m}} \rightarrow A_{n+m}$. Since $A_{n+m} \in E_{i}, E_{i_{n+m}} \rightarrow A_{n+m} \in F^{+}\left[E_{i}\right] \subseteq F^{+}\left[J^{\prime}\right]$. As the last step, we add $E_{i_{n+m}} \rightarrow A_{n+m}$ to $F^{\prime}$. Thus, $X_{n+m} \rightarrow A_{n+m}$ is not essential for adding $A_{n+m}$ to $W^{+} \cap \overline{J^{\prime}}$ and hence can be excluded.

We now have excluded one FD in $F-F^{\prime}$ that can contribute to $W^{+} \cap \overline{J^{\prime}}$. Execute this procedure repeatedly will exclude all FDs in $F-F^{\prime}$ that can contribute to $W^{+} \cap \overline{J^{\prime}}$. Eventually this procedure will halt since $F$ is finite. Thus, the proof is complete.

Lemma 4 Let $J$ be a join tree for a reduced, acyclic hypergraph $H$ and $F$ be a set of embedded FDs in $H$ such that each hyperedge of $H$ is in BCNF. Let $E_{i}$ and $E_{j}$ be two distinct nodes in $J$ such that $E_{i} \rightarrow E_{j}$. Let $P$ be the unique path between $E_{i}$ and $E_{j}$ in $J$. There exists a node $E_{k}$ on $P$ such that $E_{k} \neq E_{j}, E_{k}$ contains a key of $E_{j}$ as a subset, and $E_{i} \rightarrow E_{k}$.

Proof. Figure 12 shows the path $P$, in which we designate $E_{a}$ as the neighboring node of $E_{j}$. Let $P^{\prime}$ be the subpath of $P$ from $E_{i}$ to $E_{a}$, including $E_{i}$ and $E_{a}$. If $E_{j} \subseteq \overline{P^{\prime}}$, then by Lemma 1 , $E_{j} \subseteq E_{a}$. This means $H$ is not reduced-a contradiction. Hence, $E_{j} \not \subset \overline{P^{\prime}}$. Since $P$ is a connected subtree of $J$, by Lemma $3, F^{+}[P]$ implies the FD $E_{i} \rightarrow E_{j}$. Thus, $F^{+}[P]$ implies $E_{i} \rightarrow K$ for every key $K$ of $E_{j}$. If $\overline{P^{\prime}}$ does not contain any key of $E_{j}$ as a subset, ${\overline{P^{\prime}}}^{+}=\overline{P^{\prime}}$ where ${\overline{P^{\prime}}}^{+}$is the closure of $\overline{P^{\prime}}$ under $F^{+}[P]$. Since $E_{i} \subseteq \overline{P^{\prime}}, E_{i}^{+} \subseteq{\overline{P^{\prime}}}^{+}$. However, $E_{j} \nsubseteq \overline{P^{\prime}}\left(=\overline{P^{\prime}}\right)$ implies $E_{j} \nsubseteq E_{i}^{+}-\mathrm{a}$ contradiction. Thus, $\overline{P^{\prime}}$ contains a key $\hat{K}$ of $E_{j}$ as a subset. By Lemma $1, \hat{K} \subseteq E_{a}$. Therefore, there exists a node $E_{b}$ on $P$ such that each of the nodes in between of $E_{a}$ and $E_{b}$ on $P$, including $E_{a}$ and $E_{b}$, contains $\hat{K}$ as a subset; and every node to the left of $E_{b}$ in Figure 12, if there is any, does not contain any key of $E_{j}$. We are left to show $E_{i} \rightarrow E_{b}$. If $E_{i}$ and $E_{b}$ are the same node, we are done. Assume $E_{i} \neq E_{b}$. This implies there is an attribute $A \in\left(\hat{K}-E_{i}\right)$ that does not appear in any node to the left of $E_{b}$ on $P$. Let $P^{\prime \prime}$ be the subpath of $P$ from $E_{i}$ to $E_{b}$, including $E_{i}$ and $E_{b}$. Since $E_{i} \rightarrow K$ for every key $K$ of $E_{j}, E_{i} \rightarrow \hat{K}$. Since $P^{\prime \prime}$ is a connected subtree of $J$, by Lemma $3, F^{+}\left[P^{\prime \prime}\right]$ implies the FD $E_{i} \rightarrow \hat{K}$. Because $A$ does not appear in any node to the left of $E_{b}$, it follows that $E_{i} \rightarrow K$ where $K \rightarrow A$ is a nontrivial FD in $F^{+}\left[E_{b}\right]$. Since $E_{b}$ is in BCNF, $K \rightarrow E_{b}$ and thus $E_{i} \rightarrow E_{b}$.

\footnotetext{
${ }^{6}$ That is, $S \cup\left(X_{k} \cap \overline{J^{\prime}}\right)$ is $E_{i_{k}}$.
} 


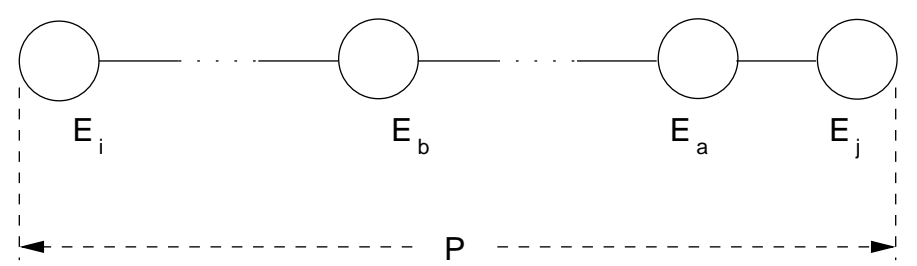

Figure 12: The Path $P$ between $E_{i}$ and $E_{j}$ of Lemma 4.

Example 13 Consider the nodes $I V_{15}$ and $B C V_{10}$ in Figure 4(b). The FDs in Figure 4(a) imply the FD $I V_{15} \rightarrow B C V_{10}$. In the path between $I V_{15}$ and $B C V_{10}$, the node $B E F I V_{12}$ contains $B$, a key of $B C V_{10}$. Also, $I V_{15} \rightarrow B E F I V_{12}$. Therefore, $B E F I V_{12}$ fits the statement of Lemma 4 .

Lemma 5 Let $J$ be a join tree for a reduced, acyclic hypergraph $H$ and $F$ be a set of embedded FDs in $H$ such that each hyperedge of $H$ is in BCNF. Let $P$ be the unique path between two distinct nodes $E_{i}$ and $E_{j}$ in $J$ where $E_{i} \rightarrow E_{j}$ and for any other node $E_{k}$ on $P$ such that $E_{k} \neq E_{i}$ and $E_{k} \neq E_{j}, E_{i} \nrightarrow E_{k}$. If $E_{j}$ is not already a neighboring node of $E_{i}$, we can rearrange the nodes on $P$ so that $E_{j}$ becomes a neighboring node of $E_{i}$.

Proof. If $E_{j}$ is already a neighboring node of $E_{i}$, then we are done. Therefore, let us assume $E_{j}$ is not a neighboring node of $E_{i}$. Like Lemma 4 , Figure 12 shows the path $P$ between $E_{i}$ and $E_{j}$ where $E_{a}$ is the designated neighboring node of $E_{j}$ on $P$. As indicated in Figure 13, we show that the edge $\left\{E_{a}, E_{j}\right\}$ can be removed, and we can add an edge between $E_{i}$ and $E_{j}$. By so doing, we obtain another join tree for $H$. We now begin our argument. Since $E_{i} \nrightarrow E_{k}$ for any other node $E_{k}$ on $P$ where $E_{k} \neq E_{i}$ and $E_{k} \neq E_{j}$, by Lemma $4, E_{i}$ contains a key $K$ of $E_{j}$ as a subset. Let $L$ be the label of the edge $\left\{E_{a}, E_{j}\right\}$. By Lemma $1, K \subseteq L$. If $K \subset L$, then since $E_{a}$ is in BCNF, $K \rightarrow E_{a}$. This means $E_{i} \rightarrow E_{a}-$ a contradiction. Thus, $L=K$. In addition, if $E_{i}$ contains an attribute $A \in\left(E_{j}-K\right)$, then by Lemma $1, A \in E_{a}$-a contradiction. Thus, we conclude that $E_{a} \cap E_{j}=E_{i} \cap E_{j}=K$. As such, we can remove the edge $\left\{E_{a}, E_{j}\right\}$ and add an edge between $E_{i}$ and $E_{j}$, as Figure 13 shows.

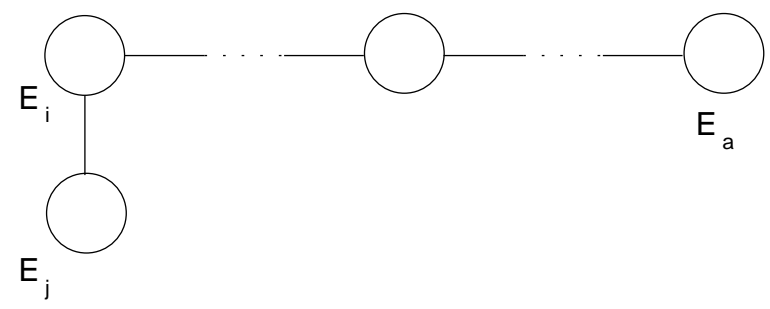

Figure 13: The Rearranged Path of Lemma 5.

Example 14 Consider the nodes $A B V_{1}$ and $B H K V_{8}$ in Figure 4(b). These two nodes fit the statement of Lemma 5, which means we can remove the edge $\left\{A B V_{1}, B D V_{5}\right\}$ and establish another 
edge between $A B V_{1}$ and $B H K V_{8}$ with exactly the same label $B$.

Theorem 1 Let $H$ be a reduced, acyclic hypergraph and $F$ be a set of embedded FDs in $H$ such that each hyperedge of $H$ is in BCNF. Let $C$ be a set of hyperedges in $H$ such that for any $E_{i}$ and $E_{j}$ in $C, E_{i} \rightarrow E_{j}$ and $E_{j} \rightarrow E_{i}$ under $F$. The hypergraph $(H-C) \cup\{\bar{C}\}$ is equivalent to $H$, and is also acyclic, and each of its hyperedges is in BCNF as well.

Proof. We first consider a simple case, which will be used later in the proof. Suppose $J$ is a join tree for $H$, and $\left\{E_{i}, E_{j}\right\}$ is an edge in $J$ such that $E_{i} \rightarrow E_{j}$ and $E_{j} \rightarrow E_{i}$ under $F$. If we create a new node $E_{i} \cup E_{j}$ and add it to $J$, and remove $E_{i}$ and $E_{j}$ from $J$, and at the same time make every

edge that was incident with $E_{i}$ or $E_{j}$ to be incident with this new node, we obtain a join tree for the hypergraph $H^{\prime}=\left(H-\left\{E_{i}, E_{j}\right\}\right) \cup\left\{E_{i} \cup E_{j}\right\}$. Hence, $H^{\prime}$ is acyclic. To show that $H^{\prime}$ is equivalent to $H$, observe that because $\left\{E_{i}, E_{j}\right\}$ is an edge in $J, E_{i} \rightarrow E_{j}$ and $E_{j} \rightarrow E_{i}$, then by Lemma 4, $E_{i}$ includes a key of $E_{j}$ and $E_{j}$ includes a key of $E_{i}$. As such, every key of $E_{i}$ implies the key of $E_{j}$ that is included in $E_{i}$. Likewise, every key of $E_{j}$ implies the key of $E_{i}$ that is included in $E_{j}$. Therefore, every key of $E_{i}$ is equivalent to every key of $E_{j}$. This means $H$ and $H^{\prime}$ are equivalent. Now suppose $X \rightarrow A$ is a nontrivial FD that holds in $E_{i} \cup E_{j}$. By Lemma $3, X \rightarrow A$ is implied by $F^{+}\left[E_{i}\right] \cup F^{+}\left[E_{j}\right]$. Since both $E_{i}$ and $E_{j}$ are in BCNF, if $X$ does not include any key of $E_{i}$ or $E_{j}$, $X=X^{+}$and $F^{+}\left[E_{i}\right] \cup F^{+}\left[E_{j}\right]$ does not imply $X \rightarrow A-$ a contradiction. Therefore, $X$ includes at least one key of $E_{i}$ or $E_{j}$. Since every key of $E_{i}$ is equivalent to every key of $E_{j}$, then $X \rightarrow E_{i}$ and $X \rightarrow E_{j}$, which implies $X \rightarrow\left(E_{i} \cup E_{j}\right)$. Thus, $E_{i} \cup E_{j}$ is in BCNF. By repeatedly applying the procedure specified in the proof for Lemma 5 and merging two functionally equivalent nodes that are neighbors, as in the case we just discussed, we can reduce the number of pairs of functionally equivalent nodes to zero. The proof is then complete.

\subsection{NNF and Connected Subtrees}

Theorem 2, the main result of this section, states that if we want to construct an NNF scheme tree that syntactically covers some hyperedges, the hyperedges must be the nodes in a connected subtree of a join tree. Otherwise, there will be a violation of NNF.

Theorem 2 Let $H$ be a reduced, acyclic hypergraph and $F$ be a set of embedded FDs in $H$ such that each hyperedge of $H$ is in BCNF and no two distinct hyperedges in $H$ are functionally equivalent. Let $T$ be an NNF scheme tree that is a syntactic cover of a set $S$ of hyperedges in $H$. The hyperedges in $S$ are precisely the nodes of a connected subtree of a join tree for $H$ (i.e., there exists a join tree $J$ for $H$ such that for any two hyperedges $E_{p}$ and $E_{q}$ in $S$, the path between $E_{p}$ and $E_{q}$ in $J$ only includes $S$ 's hyperedges).

Proof. Let us assume that $S$ 's hyperedges are not the nodes of a connected subtree of any join tree for $H$. This assumption implies that $S$ contains two distinct hyperedges $E_{p}$ and $E_{q}$ in $H$ such that the path between $E_{p}$ and $E_{q}$ in any join tree for $H$ includes some hyperedges in $H-S$. Let $J$ be a join tree for $H$ such that the path $P$ between $E_{p}$ and $E_{q}$ in $J$ is the shortest among all the possible paths between $E_{p}$ and $E_{q}$. Figure 14 shows the path $P$ and a subpath $P^{\prime}$ of $P$, where the 
endpoints of $P^{\prime}$, namely $E_{i}$ and $E_{j}$, are the only hyperedges on $P^{\prime}$ that are in $S$. Since $E_{i}$ and $E_{j}$ are the only nodes on $P^{\prime}$ that are in $S$, removing $E_{i} \cap E_{j}$ from $S$ will generate at least two connected components $C_{i}$ and $C_{j}$ where $\left(E_{i}-E_{j}\right) \subseteq C_{i}$ and $\left(E_{j}-E_{i}\right) \subseteq C_{j}$. That is, $C_{i}$ and $C_{j}$ are two connected components of the hypergraph $\left\{E-\left(E_{i} \cap E_{j}\right): E\right.$ is a hyperedge of $\left.S\right\}-\{\emptyset\}$. This means $S$ generates the nontrivial MVDs $\left(E_{i} \cap E_{j}\right) \rightarrow C_{i}$ and $\left(E_{i} \cap E_{j}\right) \rightarrow C_{j}$. Since $T$ is an NNF scheme tree that syntactically covers $S$ and $S$ generates these two MVDs on $A \operatorname{set}(T)$, by NNF's Condition 1, $M V D(T) \cup F D(T)$ implies both of these MVDs on $A \operatorname{set}(T)$. Nevertheless, if $H \cup F$ does not imply neither of them, then $T$ violates NNF's Condition 1 because $M V D(T) \cup F D(T)$ implies some MVDs on $A \operatorname{set}(T)$ that do not follow from $H \cup F$. This will give us a contradiction, which means our assumption is wrong.

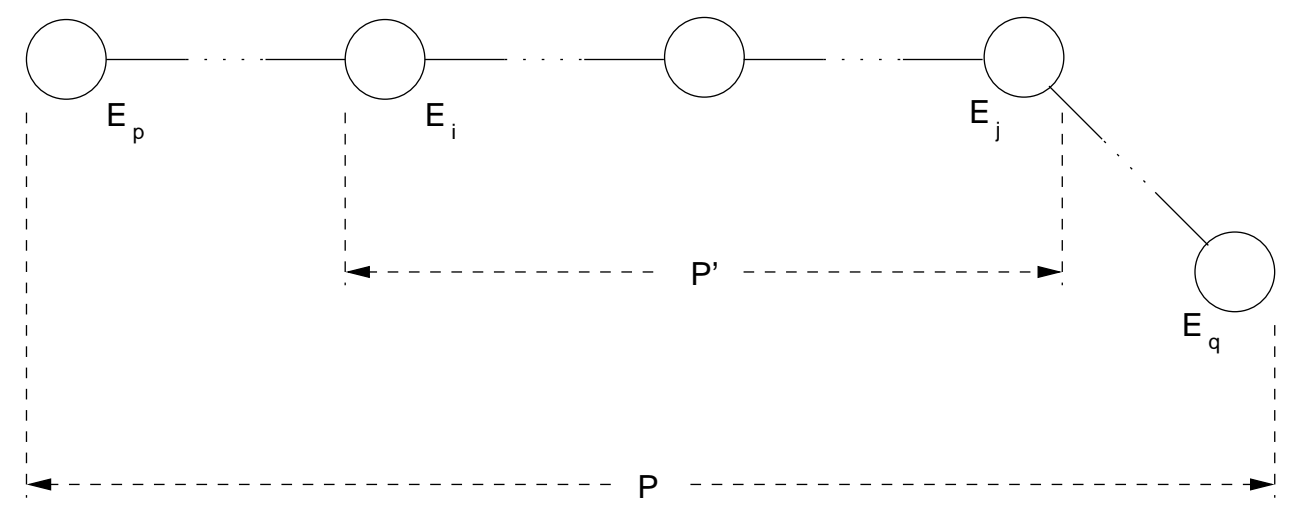

Figure 14: The Subpath $P^{\prime}$ of Theorem 2.

To show $H \cup F$ does not imply neither $\left(E_{i} \cap E_{j}\right) \rightarrow C_{i}$ nor $\left(E_{i} \cap E_{j}\right) \rightarrow C_{j}$, we first establish several claims. First, we claim that $E_{i} \cap E_{j}$ is a proper subset of every label on the path $P^{\prime}$ in Figure 14. Assume not; we derive a contradiction as follows. By Lemma $1, E_{i} \cap E_{j}$ is a subset of every label on $P^{\prime}$. Let $\left\{E_{i}^{\prime}, E_{j}^{\prime}\right\}$ be an edge on $P^{\prime}$ such that its label is equal to $E_{i} \cap E_{j}$ (i.e., $E_{i}^{\prime} \cap E_{j}^{\prime}$ $=E_{i} \cap E_{j}$ ), and as Figure 15 shows, $E_{i}^{\prime}$ and $E_{j}^{\prime}$ are chosen in such a way that $E_{i}^{\prime}$ is closer to $E_{i}$ and $E_{j}^{\prime}$ is closer to $E_{j}$. By our assumption, $P^{\prime}$ includes at least one hyperedge in $H-S$ as a node. Then, $E_{i} \neq E_{i}^{\prime}$ or $E_{j}^{\prime} \neq E_{j}$. Since $\left(E_{i}^{\prime} \cap E_{j}^{\prime}\right) \subseteq E_{i}$, if $E_{i} \neq E_{i}^{\prime}$, then we can remove the edge $\left\{E_{i}^{\prime}\right.$, $\left.E_{j}^{\prime}\right\}$ from $J$ and add an edge between $E_{i}$ and $E_{j}^{\prime}$ to obtain another join tree for $H$ with a shorter path between $E_{i}$ and $E_{j}$, and thus a shorter path for $E_{p}$ and $E_{q}$ - a contradiction. We will obtain a similar contradiction if $E_{j}^{\prime} \neq E_{j}$. Therefore, $E_{i} \cap E_{j}$ is a proper subset of every label on $P^{\prime}$. Our second claim is that $\left(E_{i} \cap E_{j}\right) \not A$ for any $A \in\left(\overline{P^{\prime}}-\left(E_{i} \cap E_{j}\right)\right)$. Assume not, then let $E$ be a node on $P^{\prime}$ that contains an attribute $A$ such that $\left(E_{i} \cap E_{j}\right) \rightarrow A$ is nontrivial. Let $E^{\prime}$ be a neighboring node of $E$ on $P^{\prime}$. By our first claim, $\left(E_{i} \cap E_{j}\right) \subset\left(E \cap E^{\prime}\right)$. Therefore, $A \in E$ and $\left(E_{i} \cap E_{j}\right) \subset E$. Since $E$ is in BCNF and $\left(E_{i} \cap E_{j}\right) \rightarrow A$ is nontrivial in $F^{+}[E],\left(E_{i} \cap E_{j}\right) \rightarrow E$. Similarly, because $E^{\prime}$ is in BCNF and $\left(E_{i} \cap E_{j}\right) \subset\left(E \cap E^{\prime}\right),\left(E_{i} \cap E_{j}\right) \rightarrow E^{\prime}$. Thus, $E$ and $E^{\prime}$ share a key of $E_{i} \cap E_{j}$ as a common key and therefore $E$ and $E^{\prime}$ are functionally equivalent-a contradiction.

Now, consider removing $\left(E_{i} \cap E_{j}\right)^{+}$, the closure of $E_{i} \cap E_{j}$ under $F$, from $H$. By our second claim, 


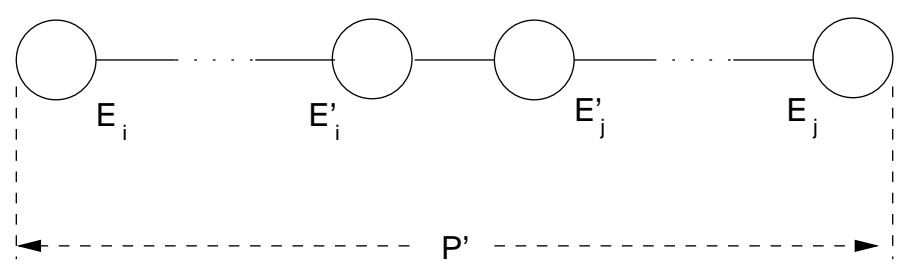

Figure 15: The Edge $\left\{E_{i}^{\prime}, E_{j}^{\prime}\right\}$ of Theorem 2 .

removing $\left(E_{i} \cap E_{j}\right)^{+}$from the nodes on $P^{\prime}$ does not remove any more attributes than removing $E_{i} \cap E_{j}$ from the nodes on $P^{\prime}$. By our first claim, all the nodes on $P^{\prime}$ remain connected after removing $E_{i} \cap E_{j}$ from the nodes on $P^{\prime}$. Thus, $E_{i}-E_{j}$ and $E_{j}-E_{i}$ are both contained as subsets in the same connected component of the hypergraph $\left\{E-\left(E_{i} \cap E_{j}\right)^{+}: E\right.$ is a hyperedge of $\left.H\right\}-$ $\{\emptyset\}$. Therefore, $H \cup F$ implies neither $\left(E_{i} \cap E_{j}\right) \rightarrow C_{i}$ nor $\left(E_{i} \cap E_{j}\right) \rightarrow C_{j}$ on $\operatorname{Aset}(T)$ and the proof is complete.

Example $15 \mathrm{~A}$ scheme tree with $B$ as the root node and $D V_{5}$ and $H K V_{8}$ as $B$ 's child nodes is not in NNF because $B D V_{5}-B H K V_{8}$ is not a connected subtree in Figure 4(b). Removing $B$ from the join tree in Figure 4(b) will not separate $D V_{5}$ and $H K V_{8}$, as the scheme tree implies.

\subsection{NNF and Critical Nodes}

Theorem 3, the main result of this section, ties critical nodes and connected subtrees together. It states that there exists an NNF scheme tree that syntactically covers the nodes in a connected subtree $S$ of a join tree if and only if $S$ does not have a critical node with respect to $S$.

Lemma 6 All join trees for a reduced, acyclic hypergraph have the same multiset of labels.

Proof. Let $H$ be an acyclic hypergraph. If $H$ has only one hyperedge, the join tree for $H$ has a single node and no label. The empty set of labels is vacuously unique. Assume this lemma is true if $H$ has $k(k \geq 1)$ or less hyperedges. Consider the case that $H$ has $k+1$ hyperedges. Since $H$ is acyclic, $H$ has a join tree $J$. Arbitrarily choose a leaf node $E_{L}$ in $J$. Since $E_{L}$ is a leaf node, removing $E_{L}$ from $J$ results in a join tree for the acyclic hypergraph $H-\left\{E_{L}\right\}$. Since $H-\left\{E_{L}\right\}$ is acyclic and has $k$ hyperedges, by the induction hypothesis, $H-\left\{E_{L}\right\}$ has a unique multiset of labels. Consider the edge that connects $E_{L}$ to another node in $J$. That edge has the label $E_{L} \cap\left(\cup_{E \in\left(H-\left\{E_{L}\right\}\right)} E\right)$, which is determined only by $E_{L}$ and $H-\left\{E_{L}\right\}$ and not by $J$. Thus, reattaching $E_{L}$ back to $J$ gives us a unique multiset of labels for $H$.

Lemma 7 Let $H$ be a reduced, acyclic hypergraph and $F$ be a set of embedded FDs in $H$ such that each hyperedge of $H$ is in BCNF and no two distinct hyperedges in $H$ are functionally equivalent. Let $\left\{L_{1}, \ldots, L_{n}\right\}$ be an equivalence class (a set) of $n \geq 1$ functionally equivalent labels of $H$. For any $i$ and $j$ such that $1 \leq i, j \leq n$ and $i \neq j, L_{i} \rightarrow L_{j}$ is nontrivial.

Proof. Assume not; we derive a contradiction as follows. First, observe that since $L_{1}, \ldots, L_{n}$ are 
labels in the same set (not multiset), $L_{1}, \ldots, L_{n}$ are all distinct. So, if there are $i$ and $j$ such that $1 \leq i, j \leq n, i \neq j$ and $L_{i} \rightarrow L_{j}$ is trivial, $L_{j} \subset L_{i}$. Since $L_{i}$ is a label, there is an edge $\left\{E_{i_{1}}, E_{i_{2}}\right\}$ in a join tree for $H$ such that $E_{i_{1}}$ and $E_{i_{2}}$ are hyperedges in $H$, and $E_{i_{1}} \cap E_{i_{2}}=L_{i}$. Since $L_{i}$ and $L_{j}$ are functionally equivalent, $L_{j} \rightarrow L_{i}$. Since $L_{j} \subset L_{i}, L_{j} \rightarrow L_{i}$, and $E_{i_{1}}$ and $E_{i_{2}}$ are both in BCNF, $E_{i_{1}}$ and $E_{i_{2}}$ share a key of $L_{j}$ as a common key. This implies $E_{i_{1}}$ and $E_{i_{2}}$ are functionally equivalent - a contradiction.

Lemma 8 Let $H$ be a reduced, acyclic hypergraph and $F$ be a set of embedded FDs in $H$ such that each hyperedge of $H$ is in BCNF and no two distinct hyperedges in $H$ are functionally equivalent. For any two distinct and functionally equivalent labels $L_{i}$ and $L_{j}$ of $H$, there is a unique hyperedge $E \in H$ such that $\left(L_{i} \cup L_{j}\right) \subseteq E$. Further, $L_{i}$ and $L_{j}$ are keys of $E$ and there is a connected subtree like the one in Figure 16(b) in any join tree for $H$.

Proof. Suppose that $H$ has no hyperedge that includes $L_{i} \cup L_{j}$ as a subset. Let $J$ be a join tree for $H$. Since $L_{i}$ and $L_{j}$ are labels of $H$, there are two nodes $E_{i}$ and $E_{j}$ in $J$ such that $L_{i} \subseteq E_{i}$ and $L_{j} \subseteq E_{j}$. By our assumption, $L_{j} \nsubseteq E_{i}$ and $L_{i} \nsubseteq E_{j}$. Without loss of generality, $E_{i}$ and $E_{j}$ are chosen in such a way that the path $P$ between them in $J$ is the shortest among all possible paths in $J$. As such, except $E_{j}$, no node on $P$ includes $L_{j}$ as a subset. Similarly, except $E_{i}$, no node on $P$ includes $L_{i}$ as a subset. Let $E_{a}$ be the neighboring node of $E_{j}$ on $P$, as Figure 16(a) shows. (It is possible that $E_{a}$ and $E_{i}$ are the same node.) Since $L_{j} \nsubseteq E_{a}$, there is an attribute $A \in L_{j}$ such that $A \notin E_{a}$. Additionally, $A$ is not in any node to the left of $E_{a}$ in Figure 16(a); otherwise by Lemma $1, A \in E_{a}$-a contradiction.

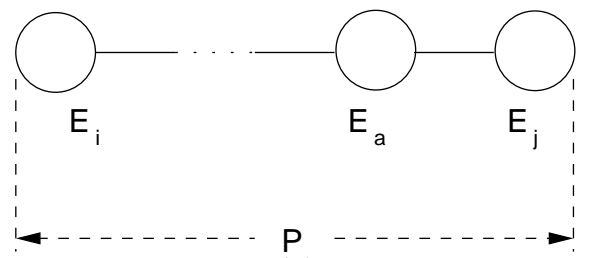

(a)

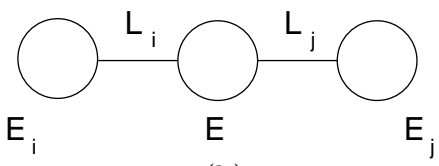

(b)

Figure 16: The Path $P$ between $E_{i}$ and $E_{j}$ and the Connected Subtree of Lemmas 8 and 9.

Since $P$ is a connected subtree of $J$, by Lemma $3, F^{+}[P]$ implies the FD $L_{i} \rightarrow L_{j}$. With respect to Figure 16(a), let $P^{\prime}$ be the subpath of $P$ from $E_{i}$ to $E_{a}$, including $E_{i}$ and $E_{a}$. Since $F^{+}[P]$ implies $L_{i} \rightarrow L_{j}$ and $A \in\left(E_{j}-\overline{P^{\prime}}\right)$, it must be that $F^{+}[P]$ implies $L_{i} \rightarrow K$ where $K \rightarrow A$ is a nontrivial FD in $F^{+}\left[E_{j}\right]$. Since $E_{j}$ is in BCNF, $K \rightarrow E_{j}$. This implies $L_{i} \rightarrow E_{j}$, which results in $E_{i} \rightarrow E_{j}$ because $L_{i} \subseteq E_{i}$. Similarly, we can show that $E_{j} \rightarrow E_{i}$. Thus, $E_{i}$ and $E_{j}$ are functionally equivalent - a contradiction.

To show that there is only one hyperedge $E \in H$ that includes $L_{i} \cup L_{j}$ as a subset, assume there are two distinct hyperedges $E_{i}$ and $E_{j}$ such that $\left(L_{i} \cup L_{j}\right) \subseteq E_{i}$ and $\left(L_{i} \cup L_{j}\right) \subseteq E_{j}$. Since $L_{i} \rightarrow L_{j}$ is nontrivial and $E_{i}$ and $E_{j}$ are both in BCNF, $L_{i} \rightarrow E_{i}$ and $L_{i} \rightarrow E_{j}$. This means $E_{i}$ and $E_{j}$ share a key of $L_{i}$ as a common key. Thus, $E_{i}$ and $E_{j}$ are two functionally equivalent hyperedges in $H-$ a 
contradiction.

We now show that there is a connected subtree like the one in Figure 16(b) in any join tree for $H$. Observe that since $L_{i}$ and $L_{j}$ are labels of $H$, there are two other hyperedges $E_{p}$ and $E_{q}$ in $H$ such that $E_{p}$ includes $L_{i}$ but not $L_{j}$ as a subset and $E_{q}$ includes $L_{j}$ but not $L_{i}$ as a subset. Let $E_{i}$ be the neighboring node of $E$ on the path between $E_{p}$ and $E$. By Lemma $1, L_{i} \subseteq\left(E_{i} \cap E\right)$. Since $L_{i} \rightarrow L_{j}$ is nontrivial, $\left(L_{i} \cup L_{j}\right) \subseteq E$, and $E$ is in BCNF, $L_{i} \rightarrow E$. Assume $L_{i} \subset\left(E_{i} \cap E\right)$. Since $E_{i}$ is also in BCNF and $L_{i} \rightarrow E, L_{i} \rightarrow E_{i}$ as well. This means $E_{i}$ and $E$ are functionally equivalent-a contradiction. Therefore, the label of the edge $\left\{E_{i}, E\right\}$ is $L_{i}$, as Figure 16(b) shows. In addition, if there exists a proper subset $L_{i}^{\prime}$ of $L_{i}$ such that $L_{i}^{\prime} \rightarrow L_{i}$, we will reach the same contradiction because $L_{i} \rightarrow E$. Therefore, $L_{i}$ is a key of $E$. The same results can be similarly established for $L_{j}$ and thus the proof is now complete.

Lemma 9 Let $H$ be a reduced, acyclic hypergraph and $F$ be a set of embedded FDs in $H$ such that each hyperedge of $H$ is in BCNF and no two distinct hyperedges in $H$ are functionally equivalent. Let $C_{i}$ and $C_{j}$ be two distinct equivalence classes of labels of $H$ such that $C_{j}$ is a parent node of $C_{i}$ in the Hasse diagram of the partial order $\succeq$ of $H$. Suppose that for each $L_{i} \in C_{i}$ and for each $L_{j} \in C_{j}, L_{j} \not L_{i}$. There exists a pair of labels $\left(L_{i}, L_{j}\right) \in C_{i} \times C_{j}$ and a unique hyperedge $E \in H$ such that $\left(L_{i} \cup L_{j}\right) \subseteq E$. Further, $L_{i}$ is a key of $E$ and there is a connected subtree like the one in Figure 16(b) in any join tree for $H$.

Proof. Let $J$ be a join tree for $H$. Assume there is no node in $J$ that includes $L_{i} \cup L_{j}$ as a subset for every pair of labels $\left(L_{i}, L_{j}\right) \in C_{i} \times C_{j}$. Choose two nodes $E_{i}$ and $E_{j}$ in $J$ such that the path $P$ between $E_{i}$ and $E_{j}$ is the shortest under the requirements that $L_{i} \subseteq E_{i}, L_{j} \subseteq E_{j}$, and $\left(L_{i}, L_{j}\right) \in C_{i} \times C_{j}$. By our assumption, except $E_{j}$, no node on $P$ includes $L_{j}$ as a subset. Similarly, except $E_{i}$, no node on $P$ includes $L_{i}$ as a subset. Let $E_{a}$ be the neighboring node of $E_{j}$ on $P$, as Figure 16(a) shows. (It is possible that $E_{a}$ and $E_{i}$ are the same node.) Since $L_{j} \nsubseteq E_{a}$, there is an attribute $A \in L_{j}$ such that $A \notin E_{a}$. Additionally, $A$ is not in any node to the left of $E_{a}$ in Figure 16(a); otherwise by Lemma $1, A \in E_{a}$-a contradiction.

Since $P$ is a connected subtree of $J$, by Lemma $3, F^{+}[P]$ implies the FD $L_{i} \rightarrow L_{j}$. With respect to Figure 16(a), let $P^{\prime}$ be the subpath of $P$ from $E_{i}$ to $E_{a}$, including $E_{i}$ and $E_{a}$. Since $F^{+}[P]$ implies $L_{i} \rightarrow L_{j}$ and $A \in\left(E_{j}-\overline{P^{\prime}}\right)$, it must be that $F^{+}[P]$ implies $L_{i} \rightarrow K$ where $K \rightarrow A$ is a nontrivial $\mathrm{FD}$ in $F^{+}\left[E_{j}\right]$. Since $E_{j}$ is in BCNF, $K \rightarrow E_{j}$. This implies $L_{i} \rightarrow E_{j}$, which means $L_{i} \rightarrow K$ for any key $K$ of $E_{j}$. If $\overline{P^{\prime}}$ does not include a key of $E_{j}$ as a subset, then ${\overline{P^{\prime}}}^{+}=\overline{P^{\prime}}$ under $F^{+}[P]$. However, since $L_{i} \subseteq \overline{P^{\prime}}$ and $L_{j} \nsubseteq \overline{P^{\prime}}$, then $F^{+}[P]$ does not imply $L_{i} \rightarrow L_{j}$-a contradiction. Therefore, $\overline{P^{\prime}}$ includes a key $\hat{K}$ of $E_{j}$ as a subset. Thus, by Lemma $1, \hat{K} \subseteq\left(E_{a} \cap E_{j}\right)$. If $\hat{K} \subset\left(E_{a} \cap E_{j}\right)$, then because $E_{a}$ is in BCNF, $E_{a}$ and $E_{j}$ share $\hat{K}$ as a common key, which means $E_{a}$ and $E_{j}$ are functionally equivalent-a contradiction. Thus, $\hat{K}=E_{a} \cap E_{j}$. Observe that $L_{j} \nrightarrow \hat{K}$; otherwise, $\hat{K} \in C_{j}$ and therefore $E_{i}$ and $E_{a}$ should have been chosen in the first place - a contradiction. Thus, $L_{i} \rightarrow \hat{K}$ where $\hat{K}$ is the label of the edge $\left\{E_{a}, E_{j}\right\}$ in Figure 16(a). In turn, $\hat{K} \rightarrow L_{j}$ and $L_{j} \nrightarrow \hat{K}$. This means $C_{j}$ is not a parent node of $C_{i}$ in the Hasse diagram of the partial order $\succeq$ of $H-$ a contradiction. Therefore, there is a pair of labels $\left(L_{i}, L_{j}\right) \in C_{i} \times C_{j}$ and a hyperedge $E \in H$ such 
that $\left(L_{i} \cup L_{j}\right) \subseteq E$.

To show that $E$ is unique, we may reuse the third paragraph of the proof for Lemma 8 . To show that $L_{i}$ is a key of $E$ and there is a connected subtree like the one in Figure 16(b) in any join tree for $H$, we may reuse the fourth paragraph of the proof for Lemma 8 for the label $L_{i}$. For the label $L_{j}$, observe that if the label of the edge $\left\{E, E_{j}\right\}$ is not $L_{j}$, then it must be a proper superset of $L_{j}$. Since $E$ and $E_{j}$ are both in BCNF, if $L_{j} \rightarrow\left(E \cap E_{j}\right)$, then $E$ and $E_{j}$ are functionally equivalent-a contradiction. Therefore, $L_{j} \not \rightarrow\left(E \cap E_{j}\right)$. Since $L_{i}$ is a key of $E, L_{i} \rightarrow\left(E \cap E_{j}\right)$. This implies $C_{j}$ is not a parent node of $C_{i}$ in the Hasse diagram of the partial order $\succeq$ of $H$-a contradiction. Thus, the label of the edge $\left\{E, E_{j}\right\}$ is $L_{j}$. The proof is now complete.

Example 16 Consider the labels $J$ and $B$ in Figure 4(b). The FD $J \rightarrow B$ is nontrivial. By Lemma 9, there is a unique node that contains $J B$ as a subset, which is $B F J V_{11}$ in Figure $4(\mathrm{~b})$.

Lemma 10 Let $H$ be a reduced, acyclic hypergraph and $F$ be a set of embedded FDs in $H$ such that each hyperedge of $H$ is in BCNF and no two distinct hyperedges in $H$ are functionally equivalent. Let $J$ be a join tree for $H$ and $S$ be a connected subtree of $J$. If there is a node in $S$ that is critical with respect to $S$, then there does not exist an NNF scheme tree that syntactically covers the set of nodes in $S$.

Proof. Suppose $T$ is an NNF scheme tree that syntactically covers the set of nodes in $S$. Let $E$ be such a critical node in $S$ and $L_{i}$ and $L_{j}$ be two labels belonged to $S$ such that $L_{i} \nrightarrow L_{j}, L_{j} \nrightarrow L_{i}$, and $\left(L_{i} \cup L_{j}\right) \subseteq E$. Since $L_{i} \nrightarrow L_{j}$ and $L_{j} \nrightarrow L_{i}, E$ must be on the path between $L_{i}$ and $L_{j}$, as Figure 17 shows. With respect to Figure 17, $L_{i} \rightarrow C_{i}$ is a hypergraph-generated MVD where $C_{i} \supseteq\left(E_{i}-L_{i}\right) \neq \emptyset$ and $C_{i}$ is a connected component of the hypergraph $\left\{E-L_{i}: E\right.$ is a hyperedge of $S\}-\{\emptyset\}$. Similarly, $L_{j} \rightarrow C_{j}$ is a hypergraph-generated MVD where $C_{j} \supseteq\left(E_{j}-L_{j}\right) \neq \emptyset$ and $C_{j}$ is a connected component of the hypergraph $\left\{E-L_{j}: E\right.$ is a hyperedge of $\left.S\right\}-\{\emptyset\}$.

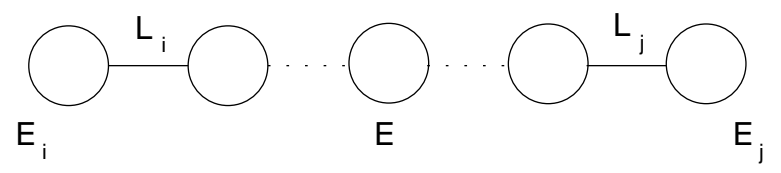

Figure 17: The Labels $L_{i}, L_{j}$, and the Critical Node $E$ of Lemma 10.

Since $T$ syntactically covers the set of nodes in $S, L_{i} \rightarrow C_{i}$ holds for $T$. Therefore, we need to test it against NNF's Condition 1, which stipulates that $M V D(T)$ and $F D(T)$ must imply $L_{i} \rightarrow C_{i}$. By Lemma 4.5 in [19] and Lemma 3 of this paper, $M V D(T)$ and $F D(T)$ imply $L_{i} \rightarrow C_{i}$ if and only if $M V D(T)$ implies $L_{i}^{+} \rightarrow C_{i}$ where $L_{i}^{+}$is the closure of $L_{i}$ under $F^{+}[S]$. Proposition 4.1 in [21] states that $M V D(T)$ is equivalent to the join dependency (JD) $\bowtie\left\{\overline{P_{1}}, \ldots, \overline{P_{n}}\right\}$ where $\overline{P_{k}}$ denotes the union of the nodes in the path $P_{k}$ of $T$ and $P_{1}, \ldots, P_{n}$ are all the paths in $T$. In addition, $L_{i}^{+} \rightarrow C_{i}$ is equivalent to the JD $\bowtie\left\{L_{i}^{+} C_{i}, L_{i}^{+}\left(\bar{S}-L_{i}^{+} C_{i}\right)\right\}$. (Note that $\bar{S}=A \operatorname{set}(T)$, the set of attributes of $T$.) Therefore, $M V D(T)$ implies $L_{i}^{+} \rightarrow C_{i}$ if and only if for every path $P_{k}$ in $T, \overline{P_{k}}$ 
$\subseteq L_{i}^{+} C_{i}$ or $\overline{P_{k}} \subseteq L_{i}^{+}\left(\bar{S}-L_{i}^{+} C_{i}\right)$ (see Chapter 8 in [16]). Likewise, $M V D(T)$ implies $L_{j}^{+} \rightarrow C_{j}$ if and only if for every path $P_{k}$ in $T, \overline{P_{k}} \subseteq L_{j}^{+} C_{j}$ or $\overline{P_{k}} \subseteq L_{j}^{+}\left(\bar{S}-L_{j}^{+} C_{j}\right)$.

We are now ready to derive a contradiction. We assume $E_{i}, E$, and $E_{j}$ each appears in (not necessarily distinct) paths $P_{i}, P$, and $P_{j}$ in $T$ respectively. Since $L_{i} \nrightarrow L_{j}$, there is an attribute $A_{j} \in L_{j}$ such that $A_{j} \notin L_{i}^{+}$. Similarly, since $L_{j} \nrightarrow L_{i}$, there is an attribute $A_{i} \in L_{i}$ such that $A_{i} \notin L_{j}^{+}$. By Lemma $1, A_{j} \notin C_{i}$; otherwise $A_{j} \in L_{i}$-a contradiction. Likewise, $A_{i} \notin C_{j}$; otherwise $A_{i} \in L_{j}$-a contradiction. Therefore, $A_{i} \notin L_{j}^{+} C_{j}$ and $A_{j} \notin L_{i}^{+} C_{i}$. Since $A_{i} \in L_{i}, A_{j} \in L_{j}$ and $\left(L_{i} \cup L_{j}\right) \subseteq E, A_{i}$ and $A_{j}$ both appear in $P$ - the path in which $E$ appears. Let $N_{i}$ and $N_{j}$ be the (not necessarily distinct) nodes in $P$ that contain $A_{i}$ and $A_{j}$ respectively. Since $L_{i} \not \rightarrow A_{j}$ and $L_{j} \nrightarrow A_{i}, L_{i} \nrightarrow N_{j}$ and $L_{j} \nrightarrow N_{i}$. Now, there are four cases to consider.

(I) $L_{i} \nrightarrow E_{i}, L_{j} \nrightarrow E_{j}$ : By NNF's Condition $1, \overline{P_{i}} \subseteq L_{i}^{+} C_{i}$ or $\overline{P_{i}} \subseteq L_{i}^{+}\left(\bar{S}-L_{i}^{+} C_{i}\right)$. Since $L_{i} \nrightarrow E_{i}$, there is an attribute $A \in E_{i}$ such that $A \notin L_{i}^{+}$. Since $\left(E_{i}-L_{i}\right) \subseteq C_{i}, A \in C_{i}$ and thus $A \notin \bar{S}-L_{i}^{+} C_{i}$. Hence, $A \notin L_{i}^{+}\left(\bar{S}-L_{i}^{+} C_{i}\right)$. Since $E_{i}$ appears in $P_{i}, A \in \overline{P_{i}}$. Thus, it must be that $\overline{P_{i}} \subseteq L_{i}^{+} C_{i}$. Likewise, $L_{j} \nrightarrow E_{j}$ implies $\overline{P_{j}} \subseteq L_{j}^{+} C_{j}$. Since $E_{i}$ and $E$ both contain $A_{i}, P_{i}$ and $P$ share $N_{i}$ as a common node. However, the node $N_{j}$ must not be a node in $P_{i}$; otherwise $A_{j} \in \overline{P_{i}}$ and $A_{j} \notin L_{i}^{+} C_{i}$ imply $\overline{P_{i}} \not \subset L_{i}^{+} C_{i}$-a contradiction. Hence, $N_{j} \neq N_{i}$ and $N_{j}$ must be lower than $N_{i}$ in $P$; otherwise $N_{j}$ is a node in $P_{i}$-a contradiction. This, however, means that $N_{i}$ is a node in $P_{j}$ because $P$ and $P_{j}$ share $N_{j}$ as a common node. This implies $A_{i} \in \overline{P_{j}}$. Nevertheless, $A_{i} \in \overline{P_{j}}$ and $A_{i} \notin L_{j}^{+} C_{j}$ imply $\overline{P_{j}} \nsubseteq L_{j}^{+} C_{j}-$ a contradiction.

(II) $L_{i} \not E_{i}, L_{j} \rightarrow E_{j}$ : Since $L_{j} \rightarrow E_{j}, L_{j}$ is a key of $E_{j}$; otherwise $E_{j}$ and its neighboring node in Figure 17 are functionally equivalent - a contradiction. Thus, with respect to Figure 17, for every $A \in\left(E_{j}-L_{j}\right), A$ does not appear in any node to the left of $E_{j}$; otherwise, by Lemma $1, A \in L_{j}$, which means $L_{j}$ is not a key of $E_{j}$-a contradiction. Therefore, $A \notin C_{i}$ for any $A \in\left(E_{j}-L_{j}\right)$ and if $L_{i} \rightarrow A$ for some $A \in\left(E_{j}-L_{j}\right)$, it must be that $L_{i} \rightarrow K$ where $K \rightarrow A$ is nontrivial in $F^{+}\left[E_{j}\right]$. Because $E_{j}$ is in BCNF, $K \rightarrow E_{j}$. This implies $L_{i} \rightarrow E_{j}$, which means $L_{i} \rightarrow L_{j}-$ a contradiction. Hence, for any $A \in\left(E_{j}-L_{j}\right), A \notin L_{i}^{+} C_{i}$.

Like in the previous case, $L_{i} \nrightarrow E_{i}$ implies $N_{i}$ is a node in $P_{j}$. For any $A \in\left(E_{j}-L_{j}\right)$, let $N$ be the node in $P_{j}$ that contains $A$. Like $A_{j}$, because $A \notin L_{i}^{+} C_{i}$ for any $A \in\left(E_{j}-L_{j}\right)$ and $N_{i}$ is a node in $P_{j}, N \neq N_{i}$ and $N$ must be lower than $N_{i}$ in $P_{j}$. Thus, for each $A \in\left(E_{j}-L_{j}\right)$, $L_{j} \nrightarrow$ Ancestor $(N)$ because $N_{i} \subseteq$ Ancestor $(N)$ and $L_{j} \nrightarrow N_{i}$. Therefore, since $L_{j} \rightarrow A$ nontrivially for each $A \in\left(E_{j}-L_{j}\right), T$ violates NNF's Condition 2-a contradiction.

(III) $L_{i} \rightarrow E_{i}, L_{j} \nrightarrow E_{j}$ : This case is symmetrical to the previous case.

(IV) $L_{i} \rightarrow E_{i}, L_{j} \rightarrow E_{j}$ : As we have already proved, $L_{j} \rightarrow E_{j}$ implies there is an attribute $A_{j}^{\prime} \in\left(E_{j}-L_{j}\right)$ such that $L_{i} \not A_{j}^{\prime}$. Likewise, $L_{i} \rightarrow E_{i}$ implies there is an attribute $A_{i}^{\prime} \in\left(E_{i}-L_{i}\right)$ such that $L_{j} \nrightarrow A_{i}^{\prime}$. Let $N_{i}^{\prime}$ and $N_{j}^{\prime}$ be the nodes in $T$ that contain $A_{i}^{\prime}$ and $A_{j}^{\prime}$ respectively. Since $L_{i} \nrightarrow A_{j}^{\prime}$ and $L_{j} \nrightarrow A_{i}^{\prime}, L_{i} \nrightarrow N_{j}^{\prime}$ and $L_{j} \not N_{i}^{\prime}$. Without loss of generality, we assume $N_{j}=N_{i}$ or $N_{j}$ is higher than $N_{i}$ in $P$. As such, $N_{j}$ is on both $P_{i}$ and $P_{j}$. Since $N_{j}$ is on $P_{i}$ and $L_{i} \rightarrow A_{i}^{\prime}$ nontrivially, $N_{i}^{\prime}$ must be higher than $N_{j}$ in $P_{i}$ because $L_{i} \nrightarrow N_{j}$; otherwise $T$ violates NNF's Condition 2-a contradiction. This implies $N_{i}^{\prime}$ is on both $P_{i}$ and $P_{j}$. Further, since $N_{i}^{\prime}$ is on $P_{j}$ 
and $L_{j} \rightarrow A_{j}^{\prime}$ nontrivially, $N_{j}^{\prime}$ must be higher than $N_{i}^{\prime}$ in $P_{j}$ because $L_{j} \nrightarrow N_{i}^{\prime}$. This also means $N_{j}^{\prime}$ is on both $P_{i}$ and $P_{j}$. Thus, $N_{i}, N_{j}, N_{i}^{\prime}, N_{j}^{\prime}$, in this order, are all on the same path. However, this will make $N_{j}^{\prime} \subseteq$ Ancestor $\left(N_{i}^{\prime}\right)$. We now have a violation of NNF's Condition 2 because $L_{i} \rightarrow A_{i}^{\prime}$ nontrivially and $L_{i} \nrightarrow$ Ancestor $\left(N_{i}^{\prime}\right)$ - a contradiction.

Lemma 11 Let $H$ be a reduced, acyclic hypergraph and $F$ be a set of embedded FDs in $H$ such that each hyperedge of $H$ is in $\mathrm{BCNF}$ and no two distinct hyperedges in $H$ are functionally equivalent. Let $J$ be a join tree for $H$ and $S$ be a connected subtree of $J$. If there is not a node in $S$ that is critical with respect to $S$, then the Hasse diagram of the partial order $\succeq$ on $S$ 's labels is a rooted tree.

Proof. For each pair of edges $\left\{E_{i}, E\right\}$ and $\left\{E, E_{j}\right\}$ in $S$, either $\left(E_{i} \cap E\right) \rightarrow\left(E \cap E_{j}\right)$ or $\left(E \cap E_{j}\right) \rightarrow$ $\left(E_{i} \cap E\right)$; otherwise $E$, a node in $S$, is critical with respect to $S$-a contradiction. Therefore, if the Hasse diagram is not a rooted tree, it must have a "V-shape." For example, there are two V-shapes in Figure 5(a). We now show that a V-shape in the Hasse diagram implies it has a critical node with respect to $S$. By this, we obtain a contradiction. Assume such a V-shape is made up by three equivalence classes $C_{i}, C_{j}$ and $C_{k}$ of functionally equivalent labels in $S$ such that $C_{i}$ and $C_{j}$ are two parent nodes of $C_{k}$ in the Hasse diagram. We have the following cases to consider.

(I) $\forall L_{i} \in C_{i} \forall L_{k} \in C_{k}\left(L_{i} \nsubseteq L_{k}\right), \forall L_{j} \in C_{j} \forall L_{k} \in C_{k}\left(L_{j} \nsubseteq L_{k}\right)$ : Since $S$ is a connected subtree, $S$ itself is also a join tree. By Lemma 9, there exists a pair of labels $\left(L_{i}, L_{k_{i}}\right) \in C_{i} \times C_{k}$ and a unique node $E_{i} \in S$ such that $\left(L_{i} \cup L_{k_{i}}\right) \subseteq E_{i}$. Further, $L_{k_{i}}$ is a key of $E_{i}$. Likewise, there exists a pair of labels $\left(L_{j}, L_{k_{j}}\right) \in C_{j} \times C_{k}$ and a unique node $E_{j} \in S$ such that $\left(L_{j} \cup L_{k_{j}}\right) \subseteq E_{j}$. Further, $L_{k_{j}}$ is a key of $E_{j}$. If $E_{i} \neq E_{j}$, then because $L_{k_{i}}$ and $L_{k_{j}}$ are functionally equivalent and $L_{k_{i}}$ and $L_{k_{j}}$ are keys of $E_{i}$ and $E_{j}$ respectively, $E_{i}$ and $E_{j}$ are functionally equivalent-a contradiction. Hence, $E_{i}=E_{j}$ and $E_{i}$ is a critical node.

(II) $\forall L_{i} \in C_{i} \forall L_{k} \in C_{k}\left(L_{i} \nsubseteq L_{k}\right), \exists L_{j} \in C_{j} \exists L_{k_{j}} \in C_{k}\left(L_{j} \subset L_{k_{j}}\right)$ : By Lemma 9 , there exists a pair of labels $\left(L_{i}, L_{k_{i}}\right) \in C_{i} \times C_{k}$ and a unique node $E_{i} \in S$ such that $\left(L_{i} \cup L_{k_{i}}\right) \subseteq E_{i}$. Further, $L_{k_{i}}$ is a key of $E_{i}$. If $L_{k_{j}}=L_{k_{i}}$, then $E_{i}$ is a critical node. Assume $L_{k_{j}} \neq L_{k_{i}}$. By Lemma 8, there is a node $E_{k}$ of which $L_{k_{i}}$ and $L_{k_{j}}$ are keys. If $E_{i} \neq E_{k}$, then since $L_{k_{i}}$ is a key for both of them, $E_{i}$ and $E_{k}$ are functionally equivalent-a contradiction. Hence, $E_{i}=E_{k}$ and thus $L_{j} \subset L_{k_{j}} \subset E_{i}$. Hence, $E_{i}$ is a critical node.

(III) $\exists L_{i} \in C_{i} \exists L_{k_{i}} \in C_{k}\left(L_{i} \subset L_{k_{i}}\right), \forall L_{j} \in C_{j} \forall L_{k} \in C_{k}\left(L_{j} \not L_{k}\right)$ : This case is symmetrical to the previous case.

(IV) $\exists L_{i} \in C_{i} \exists L_{k_{i}} \in C_{k}\left(L_{i} \subset L_{k_{i}}\right), \exists L_{j} \in C_{j} \exists L_{k_{j}} \in C_{k}\left(L_{j} \subset L_{k_{j}}\right):$ If $L_{k_{i}}=L_{k_{j}}$, then either one of the two nodes of an edge whose label is $L_{k_{i}}$ is a critical node. If $L_{k_{i}} \neq L_{k_{j}}$, then by Lemma 8, there is a node $E_{k}$ of which $L_{k_{i}}$ and $L_{k_{j}}$ are keys. Hence, $E_{k}$ is a critical node.

Lemma 12 Let $H$ be a reduced, acyclic hypergraph and $F$ be a set of embedded FDs in $H$ such that each hyperedge of $H$ is in BCNF and no two distinct hyperedges in $H$ are functionally equivalent. Let $J$ be a join tree for $H$ and $S$ be a connected subtree of $J$. If there is not a node in $S$ that is critical with respect to $S$, then there exists an NNF scheme tree that syntactically covers 
the hyperedges in $S$.

Proof. By Lemma 11, the Hasse diagram of the partial order $\succeq$ on $S$ 's labels is a rooted tree $T$. Suppose Step 2 of Procedure AttachHyperedges finds two nodes $N_{i}$ and $N_{j}$ in different paths of $T$ for a node $E$ in $S$. Thus, there are labels $L_{i} \in N_{i}$ and $L_{j} \in N_{j}$ such that $\left(L_{i} \cup L_{j}\right) \subseteq E$. Since $N_{i}$ and $N_{j}$ are in different paths of $T, L_{i} \nrightarrow L_{j}$ and $L_{j} \nrightarrow L_{i}$. This implies $E$ is critical-a contradiction. Hence, we may run Steps 2, 3 and 4 of Procedure AttachHyperedges on $T$ to obtain a scheme tree $T^{\prime}$.

We first prove by induction on the number $n$ of nodes in $T$ that every node in $S$ appears in a path of $T^{\prime}$. If $n=0$, then $T$ is empty. This implies $S$ has zero or one node. In the former case, our claim is vacuously true. In the latter case, the only node of $S$ becomes the only node of $T^{\prime}$. If $n=1, T$ has a single node. Then, all the labels in that node are merged together to form the root node of $T^{\prime}$ and each node in $S$ forms a path in $T^{\prime}$. Therefore, our claim is also true when $n=1$. Assume our claim is true if $n \leq k$ for some $k \geq 1$. Run Procedure MoveLabelsToCenterNodes on $S$. Let $T_{k}$ be an NNF skeleton with $k$ nodes. Consider a child node $N_{c}$ of a node $N_{p}$ in the Hasse diagram of $\succeq$ where $N_{p}$ is already a node in $T_{k}$. We obtain NNF skeleton $T_{k+1}$ by adding $N_{c}$ as a child node to $N_{p}$ in $T_{k}$. If $N_{c} \succeq N_{p}$ nontrivially, then by Lemma 9 there are labels $L_{p} \in N_{p}$, $L_{c} \in N_{c}$, and a unique node $E$ in $S$ such that $\left(L_{p} \cup L_{c}\right) \subseteq E$. If $N_{c} \succeq N_{p}$ trivially, then there are labels $L_{p} \in N_{p}, L_{c} \in N_{c}$ such that $L_{p} \subset L_{c}$. Let $E^{\prime}$ be the node of an edge with the label $L_{p}$ such that if that edge was removed from $S, E^{\prime}$ would separate from $L_{c}$. Observe that for each $L \in N_{c}$, $L \nsubseteq E^{\prime}$. Thus, $E^{\prime}$ must be attached to $N_{p}$. By the induction hypothesis, $L_{p} \subseteq$ Ancestor $\left(N_{p}\right)$ in $T^{\prime}$. By Lemma 1, the intersection of a label in $N_{p}$ and a label in $N_{c}$ is a subset of $L_{p}$. Therefore, since $N_{c}$ is a child node of $N_{p}$ in $T$, every label in $N_{c}$ is a subset of Ancestor $\left(N_{c}\right)$ in $T^{\prime}$. This means that every node in $S$ that is attached to $N_{c}$ appears in a path of $T^{\prime}$. The induction step is thus complete. Since we do not add any attribute to $T$ that is not in any node in $S, A \operatorname{set}\left(T^{\prime}\right)=\bar{S}$. Hence, $T^{\prime}$ syntactically covers the set of nodes in $S$.

We are left to prove that $T^{\prime}$ is in NNF. Since $S$ is a connected subtree of $J, S$ itself is also a join tree. Thus, the set of MVDs generated by $S$ is equivalent to $\bowtie\left\{E_{1}, \ldots, E_{m}\right\}$ where $E_{1}, \ldots, E_{m}$ are the nodes in $S$ [3]. Hence, to prove $T^{\prime}$ satisfies NNF's Condition 1, we need to show that $M V D\left(T^{\prime}\right)$ and $F D\left(T^{\prime}\right)$ are equivalent to $\bowtie\left\{E_{1}, \ldots, E_{m}\right\}$ and $F^{+}[S]$. We stated earlier that $M V D\left(T^{\prime}\right)$ is equivalent to $\bowtie\left\{\overline{P_{1}}, \ldots, \overline{P_{n}}\right\}$ where $P_{1}, \ldots, P_{n}$ are all the paths in $T^{\prime}$ (see the proof for Lemma 10). Also, observe that $F D\left(T^{\prime}\right)$ is equivalent to $F^{+}[S]$. Thus, one direction of the equivalence is easily established because $T^{\prime}$ syntactically covers the set of nodes in $S$. For each path $P$ in $T^{\prime}$, consider $P$ 's leaf node $N_{E}=\{A \in E: A$ does not appear in any label in any node of $T\}$ for some hyperedge $E \in S$. Let $N_{E}$ 's parent node be $N$. Since $E$ contains a label in $N, E \rightarrow$ Ancestor $(N)$ in $T^{\prime}$. Therefore, $\bar{P} \subseteq E^{+}$. By Chapter 8 in [16], $T^{\prime}$ satisfies NNF's Condition 1.

To prove $T^{\prime}$ satisfies NNF's Condition 2, observe that by Lemma 3 it is sufficient to only consider $F^{+}[S]$. Thus, let $X \rightarrow A$ be a nontrivial FD in $F^{+}[E]$ for some node $E$ in $S$. Since $E$ is in BCNF, $X \rightarrow E$. Assume $E$ is attached to a node $N$ in $T$. It is clear that $E \rightarrow$ Ancestor $(N)$ in $T^{\prime}$ and thus $X \rightarrow E \cup \operatorname{Ancestor}(N)$ in $T^{\prime}$. It follows that $T^{\prime}$ satisfies NNF's Condition 2 as well. 
Theorem 3 Let $H$ be a reduced, acyclic hypergraph and $F$ be a set of embedded FDs in $H$ such that each hyperedge of $H$ is in BCNF and no two distinct hyperedges in $H$ are functionally equivalent. Let $J$ be a join tree for $H$ and $S$ be a connected subtree of $J$. There exists an NNF scheme tree that syntactically covers the hyperedges in $S$ if and only if there is not a node in $S$ that is critical with respect to $S$.

Proof. This theorem follows immediately from Lemmas 10, 11 and 12.

\subsection{Correctness}

Theorem 4 Procedure Main of Section 3.1 generates a largest NNF scheme tree from its input in polynomial time.

Proof. Let $T$ and $J$ respectively be the input NNF skeleton and the input modified join tree of Procedure AttachHyperedges. We first show that the set $S$ defined in Step 1 of Procedure AttachHyperedges constitutes a connected subtree of $J$ and it does not have a critical node. By Lemmas 7, 8, and 9, if $N_{c} \succeq N_{p}$ nontrivially for two nodes $N_{p}$ and $N_{c}$ in $T$ where $N_{p}$ is the parent of $N_{c}$, then the edges whose labels are in $N_{p}$ and $N_{c}$ clearly form a connected subtree of $J$. On the other hand, if $N_{c} \succeq N_{p}$ trivially, then there are labels $L_{p} \in N_{p}$ and $L_{c} \in N_{c}$ such that $L_{p} \subset L_{c}$. As such, we may make an edge with the label $L_{p}$ to be incident with the center node of $N_{c}$ in $S$. Thus, the edges whose labels are in $N_{p}$ and $N_{c}$ also form a connected subtree of $J$.

We now proceed to prove that $S$ does not have a critical node. Assume not, let $L_{i}$ and $L_{j}$ be two labels in $T$ such that $L_{i} \nrightarrow L_{j}$ and $L_{j} \nrightarrow L_{i}$; and let $E$ be a node in $S$ such that $\left(L_{i} \cup L_{j}\right) \subseteq E$. As such, $E$ must be on the path between $L_{i}$ and $L_{j}$ in $S$, as Figure 17 shows. If there is at least one label $L_{k}$ between $L_{i}$ and $L_{j}$ on that path such that $L_{k} \nrightarrow L_{i}$ and $L_{k} \nrightarrow L_{j}$, then the existence of $E$ will lead to $L_{k} \rightarrow L_{i}$ or $L_{k} \rightarrow L_{j}$-a contradiction. Let $L_{i} \in N_{i}$ and $L_{j} \in N_{j}$ and assume $N_{i}$ and $N_{j}$ are nodes in $T$ that have different parents. Then, there is at least one label $L_{k}$ between $L_{i}$ and $L_{j}$ in $S$ such that $L_{k} \nrightarrow L_{i}$ and $L_{k} \nrightarrow L_{j}$-a contradiction. Hence, $N_{i}$ and $N_{j}$ are child nodes of the same parent $N_{k}$ in $T$. As such, $N_{i} \nsucceq N_{j}, N_{j} \nsucceq N_{i}, N_{k} \nsucceq N_{i}$, and $N_{k} \nsucceq N_{j}$. Since $N_{k}$ is the parent of $N_{i}$ and $N_{j}$ in $T$, there are nodes $E_{i}$ and $E_{j}$ in $S$ such that $\left(L_{k_{i}} \cup L_{i}\right) \subseteq E_{i}$ and $\left(L_{k_{j}} \cup L_{j}\right) \subseteq E_{j}$ where $L_{k_{i}}, L_{k_{j}} \in N_{k}, L_{i} \in N_{i}$ and $L_{j} \in N_{j}$. We now have the following cases to consider.

(I) $N_{i} \succeq N_{k}$ nontrivially and $N_{j} \succeq N_{k}$ nontrivially: Assume $E_{i}=E_{j}$. By Lemma $9, L_{i}$ and $L_{j}$ are keys of $E_{i}$. This implies $N_{i} \succeq N_{j}$ and $N_{j} \succeq N_{i}$-a contradiction. Hence, $E_{i} \neq E_{j}$. As such, there is a label $L_{k} \in N_{k}$ that is in between of $L_{i}$ and $L_{j}$ in $S$ - a contradiction. Hence, there is no node in $S$ that is critical.

(II) $N_{i} \succeq N_{k}$ nontrivially and $N_{j} \succeq N_{k}$ trivially: Assume $E_{i}=E_{j}$. By Lemma $9, L_{i}$ is a key of $E_{i}$. This implies $N_{i} \succeq N_{j}$-a contradiction. Hence, $E_{i} \neq E_{j}$. We may now proceed like in the previous case from this point on.

(III) $N_{i} \succeq N_{k}$ trivially and $N_{j} \succeq N_{k}$ nontrivially: This case is symmetrical to the previous case. (IV) $N_{i} \succeq N_{k}$ trivially and $N_{j} \succeq N_{k}$ trivially: Suppose there is a label $L_{k} \in N_{k}$ in between of $N_{i}$ 's center node and $N_{j}$ 's center node. Then, there is a label $L_{k} \in N_{k}$ in between of $L_{i}$ and $L_{j}$ in $S$-a 
contradiction. Hence, there is not a label $L_{k} \in N_{k}$ in between of $N_{i}$ 's center node and $N_{j}$ 's center node. However, in this case Procedure CalculateLabelCnt at best selects one of $N_{i}$ and $N_{j}$ or at worst selects none of $N_{i}$ and $N_{j}$ in constructing a largest NNF skeleton. Thus, $S$ has no critical nodes.

To prove that Procedure Main generates a largest NNF scheme tree, we show that if we add one more node (hyperedge) in $J$ to $S, S$ will have a critical node. Now, suppose we add one more equivalence class $C$ of labels in the Hasse diagram of $\succeq$ to $T$. Because of Theorem 2, $C$ must be connected to an equivalence class $C_{T}$ already in $T$. Further, $C$ cannot be a child node of $C_{T}$ in the Hasse diagram of $\succeq$ (i.e., $C \nsucceq C_{T}$ ); otherwise, Procedure CalculateLabelCnt has already considered $C$ in constructing $T$. Suppose $C_{T} \nsucceq C$. If the label of the edge between $C$ 's center node and $C_{T}$ 's center node is in $C$, then $C_{T}$ 's center node is a critical node. If the label of the edge between $C$ 's center node and $C_{T}$ 's center node is in $C_{T}$, then $C$ 's center node is a critical node. Now suppose $C_{T} \succeq C$. Observe that $C_{T}$ cannot be a root node in the Hasse diagram of $\succeq$; otherwise $C_{T} \nsucceq C$. Then, there is a $\mathrm{V}$-shape in $T$, which means $C_{T}$ 's center node is a critical node.

\subsection{Complexity Analysis}

We now prove by a worst-case analysis that Procedure Main runs in time polynomial in the size of the input. We first consider the two preparatory procedures: Procedure MergeHyperedges and Procedure CreateJoinTree. Procedure MergeHyperedges uses Algorithm 4.4 on page 66 in [16] in its computation. This algorithm has time complexity $O(p)$, where $p$ is the number of symbols required to represent the given set of FDs. Thus, generating the closure $E^{+}$of one hyperedge $E$ takes $O(p)$ time. For $q \geq 1$ hyperedges, it takes $O(p q)$ time to compute the $p$ closures of the $q$ hyperedges. Let $n$ be the number of symbols required to represent the input acyclic hypergraph and the set of embedded FDs. It is easy to see that $p$ and $q$ are proportional to $n$. Hence, it takes $O\left(n^{2}\right)$ time to compute the $q$ closures. Now, consider merging two hyperedges when their closures are equal. Given $q>1$ closures over $r>1$ distinct attributes, we compute the number of comparisons in the worst case that no pair of closures is equal. First, we use a matrix with $q$ rows and $r$ columns to represent these $q$ closures where $\operatorname{cell}(i, j)$ - the cell at row $i$ and column $j$-is equal to 1 if closure $C_{i}$ has attribute $A_{j}$; otherwise, $\operatorname{cell}(i, j)$ is equal to 0 . Filling up this matrix obviously takes $O(n)$ time. With this matrix, closure $C_{i}$ is equal to closure $C_{j}$ if and only if $\operatorname{cell}(i, 1)=\operatorname{cell}(j, 1), \operatorname{cell}(i, 2)=\operatorname{cell}(j, 2), \ldots$, and $\operatorname{cell}(i, r)=\operatorname{cell}(j, r)$. Thus, checking whether $C_{i}=C_{j}$ takes $r$ comparisons. Proving closure $C_{1}$ is not equal to any other closure therefore takes $(q-1) r$ comparisons. For closure $C_{2}$, it similarly takes $(q-2) r$ comparisons. The same reasoning applies to all the other closures. Hence, it takes $(q-1) r+(q-2) r+\cdots+r=q(q-1) r / 2$ comparisons to prove that no closure is equal to another closure. Since $r$ is also proportional to $n$, it takes $O\left(n^{3}\right)$ time to show that no pair of closures is equal. As stated in [24], a straightforward implementation for Procedure CreateJoinTree runs in time quadratic in the size of the input acyclic hypergraph. Hence, both Procedure MergeHyperedges and Procedure CreateJoinTree run in polynomial time 
with respect to $n$.

As for Procedure ConstructHasseDiagram0f $\succeq$, Procedure MoveLabelsToCenterNodes, and Procedure ExtractLargestNNFSkeleton, our experiments strongly indicate that these three procedures considered as a whole run in time quadratic in the number of hyperedges. However, since the hyperedges and equivalence classes of labels are generated randomly, this can only be considered as an average-case complexity. For a worst-case analysis of Procedure ConstructHasseDiagram0f $\succeq$, let $n$ be the number of symbols required to represent the input acyclic hypergraph and the set of embedded FDs. Observe that the number of labels is one less than the number of nodes (hyperedges) in any join tree. Hence, sorting functionally equivalent labels in a join tree into equivalence classes is similar to merging functionally equivalent hyperedges in an acyclic hypergraph. Further, for two distinct labels $L_{i}$ and $L_{j}, L_{i}^{+} \subset L_{j}^{+}, L_{j}^{+} \subset L_{i}^{+}$, and $L_{i}^{+}=L_{j}^{+}$can all be tested successively in the same pass. Thus, sorting labels into equivalence classes and generating the partial order $\succeq$ can be done at the same time. Therefore, Procedure ConstructHasseDiagramOf $\succeq$ at most takes $O\left(n^{3}\right)$ time. Procedure MoveLabelsToCenterNodes at most reorganizes every edge in a join tree once. Thus, Procedure MoveLabelsToCenterNodes runs in time linear in the number of labels in a join tree, which is proportional to $n$. The time complexity of Procedure ExtractLargestNNFSkeleton clearly depends on the time complexity of Procedure CalculateLabelCnt, which is recursive. This recursive procedure visits each equivalence class of labels in the Hasse diagram of $\succeq$ once as it calculates its labelCnt. Hence, Procedure CalculateLabelCnt runs in time linear in the number of equivalence classes of labels, which again is proportional to $n$. Obviously, each other step of Procedure ExtractLargestNNFSkeleton has time complexity $O(n)$. Hence, Procedure ExtractLargestNNFSkeleton has time complexity $O(n)$. For Procedure AttachHyperedges, note that a reasonable implementation of a label has two pointers that point at the two nodes (hyperedges) to which it connects. Thus, given an NNF skeleton, finding the set $S$ of Step 1 in Procedure AttachHyperedges takes time linear in the number of labels in the NNF skeleton. Attaching them to the NNF skeleton then also takes time linear in the number of labels in the skeleton, which is proportional to $n$.

\section{Concluding Remarks}

In this paper we presented a polynomial-time algorithm to generate a largest redundancy-free XML storage structure from an acyclic hypergraph and a set of embedded FDs where each hyperedge is in BCNF. The algorithm generates a largest NNF scheme tree, which can then be mapped to a redundancy-free XML storage structure. Besides reducing space requirements and overcoming update anomalies, the algorithm also determines a largest set of hyperedges such that no join is needed to navigate from one data item to another within the storage structure. Further, when applied repeatedly on hypergraph edges not already included in generated scheme-trees, the algorithm always yields redundancy-free XML storage structures and often, especially in practical cases, yields the fewest. This, then, also reduces the join cost to navigate from any data item within the application to any other. 
It is an open problem to determine whether a polynomial-time algorithm exists to generate a minimum number of scheme trees from an acyclic hypergraph and a set of embedded FDs where each hyperedge is in BCNF. However, since NNF is equivalent to BCNF when only flat relation schemes are allowed [19], BCNF's well-known intractable problems might carry over to this open problem. For example, Theorem 4.22 in [14] states that "The problem of finding a lossless join and nonredundant decomposition of schema $R$ that is in BCNF with respect to a set $F$ of FDs over $R$, and such that the number of relation schemas in $R$ is less than or equal to some natural number $k \geq 1$ is NP-hard." It suffices to say that at this point more research is needed for this open problem.

\section{Acknowledgements}

The work described in this paper was partially supported by Strategic Research Grants 7001945 and 7002140 of City University of Hong Kong. D.W. Embley was supported in part by the National Science Foundation under grant numbers 0083127 and 0414644.

\section{References}

[1] Reema Al-Kamha. Conceptual XML for Systems Analysis. PhD dissertation, Department of Computer Science, Brigham Young University, Provo, Utah, June 2007.

[2] Marcelo Arenas and Leonid Libkin. A normal form for XML documents. ACM Transactions on Database Systems, 29:195-232, 2004.

[3] Catriel Beeri, Ronald Fagin, David Maier, and Mihalis Yannakakis. On the desirability of acyclic database schemes. Journal of the ACM, 30(3):479-513, 1983.

[4] Ronald Bourret. XML and databases. 2005. http://www.rpbourret.com/xml/XMLAndDatabases.htm.

[5] Ronald Bourret. XML database products. 2007. http://www.rpbourret.com/xml/XMLDatabaseProds.htm.

[6] Yi Chen, Susan B. Davidson, Carmem S. Hara, and Yifeng Zheng. RRXF: Redundancy reducing XML storage in relations. In Proceedings of 29th International Conference on Very Large Data Bases, pages 189-200, Berlin, Germany, September 9-12 2003.

[7] David W. Embley and Wai Yin Mok. Developing XML documents with guaranteed "good" properties. In Proceedings of the 20th International Conference on Conceptual Modeling, pages 426-441, Yokohama, Japan, November 27-30 2001.

[8] Ronald Fagin, Alberto O. Mendelzon, and Jeffrey D. Ullman. A simplified universal relation assumption and its properties. ACM Transactions on Database Systems, 7(3):343-360, 1982. 
[9] Thorsten Fiebig, Sven Helmer, Carl-Christian Kanne, Guido Moerkotte, Julia Neumann, Robert Schiele, and Till Westmann. Anatomy of a native XML base management system. The VLDB Journal, 11(4):292-314, 2002.

[10] Nathan Goodman, Oded Shmueli, and Y. C. Tay. GYO reductions, canonical connections, tree and cyclic schemas and tree projections. In Proceedings of the Second ACM SIGACT-SIGMOD Symposium on Principles of Database Systems, pages 267-278, Atlanta, Georgia, March 21-23 1983.

[11] Gang Gou and Rada Chirkova. Efficiently querying large XML data repositories: A survey. IEEE Transactions on Knowledge and Data Engineering, 19(10):1381-1403, 2007.

[12] H. V. Jagadish, Shurug Al-Khalifa, Adriane Chapman, Laks V. S. Lakshmanan, Andrew Nierman, Stelios Paparizos, Jignesh M. Patel, Divesh Srivastava, Nuwee Wiwatwattana, Yuqing $\mathrm{Wu}$, and Cong Yu. TIMBER: A native XML database. The VLDB Journal, 11(4):274-291, 2002 .

[13] Solmaz Kolahi and Leonid Libkin. XML design for relational storage. In Proceedings of the 16th International Conference on World Wide Web, pages 1083-1092, Banff, Alberta, Canada, May 8-12 2007.

[14] Mark Levene and George Loizou. A Guided Tour of Relational Databases and Beyond. Springer, 1999.

[15] Leonid Libkin. Normalization theory for XML. In Proceedings of the 5th International XML Database Symposium, pages 1-13, Vienna, Austria, September 23-24 2007.

[16] David Maier. The Theory of Relational Databases. Computer Science Press, 1983.

[17] Wai Yin Mok. A comparative study of various nested normal forms. IEEE Transactions on Knowledge and Data Engineering, 14(2):369-385, 2002.

[18] Wai Yin Mok and David W. Embley. Generating compact redundancy-free XML documents from conceptual-model hypergraphs. IEEE Transactions on Knowledge and Data Engineering, 18(8):1082-1096, 2006.

[19] Wai Yin Mok, Yiu-Kai Ng, and David W. Embley. A normal form for precisely characterizing redundancy in nested relations. ACM Transactions on Database Systems, 21(1):77-106, 1996.

[20] Matthias Nicola and Bert Van der Linden. Native XML support in DB2 universal database. In Proceedings of the 31st International Conference on Very Large Data Bases, pages 1164-1174, Trondheim, Norway, August 30 - September 22005.

[21] Z. Meral Özsoyoglu and Li-Yan Yuan. A new normal form for nested relations. ACM Transactions on Database Systems, 12(1):111-136, 1987. 
[22] Klaus-Dieter Schewe. Redundancy, dependencies and normal forms for XML databases. In Proceedings of the Sixteenth Australasian Database Conference, pages 7-16, Newcastle, Australia, January 31st - February 3rd 2005.

[23] Bart Steegmans, Ronald Bourret, Owen Cline, Olivier Guyennet, Shrinivas Kulkarni, Stephen Priestley, Valeriy Sylenko, and Ueli Wahli. XML for DB2 Information Integration. IBM, July 2004.

[24] Robert Endre Tarjan and Mihalis Yannakakis. Simple linear-time algorithms to test chordality of graphs, test acyclicity of hypergraphs, and selectively reduce acyclic hypergraphs. SIAM Journal on Computing, 13(3):566-579, 1984.

[25] Millist W. Vincent, Jixue Liu, and Chengfei Liu. Strong functional dependencies and their application to normal forms in XML. ACM Transactions on Database Systems, 29(3):445-462, 2004.

[26] Junhu Wang and Rodney W. Topor. Removing XML data redundancies using functional and equality-generating dependencies. In Proceedings of the Sixteenth Australasian Database Conference, pages 65-74, Newcastle, Australia, January 31st - February 3rd 2005.

[27] Cong Yu and H. V. Jagadish. XML schema refinement through redundancy detection and normalization. The VLDB Journal, 17(2):203-223, 2008. 